\title{
Linking inhibitory control to math achievement via comparison of conflicting decimal numbers
}

\author{
Linsah Coulanges ${ }^{1 *}$, Roberto A. Abreu-Mendoza ${ }^{1}$, Sashank Varma ${ }^{2}$, Melina Uncapher ${ }^{3}$, Adam \\ Gazzaley $^{3}$, Joaquin Anguera ${ }^{3}$, and Miriam Rosenberg-Lee ${ }^{1^{*}}$ \\ ${ }^{1}$ Department of Psychology, Rutgers University - Newark \\ ${ }^{2}$ Department of Educational Psychology, University of Minnesota \\ ${ }^{3}$ Neuroscape, Department of Neurology, Weill Institute for the Neurosciences, University of \\ California San Francisco. \\ *Corresponding Author
}

\begin{abstract}
Author Note
Linsah Coulanges is now at the Department of Psychology, University of Pittsburgh, USA
\end{abstract}




\begin{abstract}
The relationship between executive functions (EF) and academic achievement is wellestablished, but leveraging this insight to improve educational outcomes remains elusive. Here, we propose a framework for relating the role of specific EF on specific precursor skills that support later academic learning. Starting from the premise that executive functions contribute to general math skills both directly - supporting the execution of problem solving strategies - and indirectly - supporting the acquisition of precursor mathematical content, we hypothesize that the contribution of domain-general EF capacities to precursor skills that support later learning can help explain relations between EF and overall math skills. We test this hypothesis by examining whether the contribution of inhibitory control on general math knowledge can be explained by inhibition's contribution to processing rational number pairs that conflict with individual's prior whole number knowledge. In 97 college students (79 female, age $=20.58$ years), we collected three measures of EF: working memory (backwards spatial span), inhibition (color-word Stroop) and cognitive flexibility (task switching), and timed and untimed standardized measures of math achievement. Our target precursor skill was a decimals comparison task where correct responses were inconsistent with prior whole number knowledge (e.g., 0.27 vs. 0.9). Participants performed worse on these trials relative to the consistent decimals pairs (e.g., 0.2 vs. 0.87). Individual differences in the Stroop task predicted performance on inconsistent decimal comparisons, which in turn predicted general math achievement. With respect to relating inhibitory control to math achievement, Stroop performance was an independent predictor of achievement after accounting for age, working memory and cognitive flexibility, but decimal performance mediated this relationship. Finally, we found inconsistent decimals performance mediated the relationship of inhibition with rational number performance, but not other advanced mathematical concepts. These results pinpoint the specific contribution of inhibitory control to rational number understanding, and more broadly are consistent with the hypothesis that acquisition of foundational mathematical content can explain the relationships between executive functions and academic outcomes, making them promising targets for intervention.
\end{abstract}

Keywords: Inhibitory control; Rational numbers; Decimals; Executive functions; Mathematics achievement; Individual differences 


\section{Introduction}

Robust evidence for relations between executive functions (EF) and academic achievement has accumulated for decades (Diamond, 2013; Peng, Namkung, Barnes, \& Sun, 2016; Zelazo, Blair, \& Willoughby, 2017). Yet, the practical utility of this insight has been limited: efforts to train executive functions in order to improve educational outcomes have largely failed (Melby-Lervag \& Hulme, 2013). A separate line of research has focused on identifying early domain specific skills and capacities, which predict later achievement. By analogy to the role of phonological awareness in reading, tasks like nonsymbolic and symbolic comparison and estimation have been proposed as precursor skills in mathematics (De Smedt, Noël, Gilmore, \& Ansari, 2013; Gersten \& Chard, 1999; Halberda, Mazzocco, \& Feigenson, 2008; Sasanguie, Lyons, De Smedt, \& Reynvoet, 2017; Siegler \& Booth, 2004). Here, we define a precursor skill as a lowerlevel math task that is thought to index understanding of a foundational mathematical concept.

When studies of precursor skills consider the effects of domain general skills like EF, they typically report one of two results: either (1) the relationship between the skill and math achievement remains significant after including the EF measure, such as fraction arithmetic predicts later math achievement after controlling for working memory (e.g., Siegler et al., 2012); (2) or EF explains the relationship between the precursor skill and math achievement and the precursor skill is no longer significant, such as inhibitory control explaining the contribution of nonsymbolic dot comparison tasks to math achievement (e.g., Gilmore et al., 2013). This all-ornone approach overlooks the fact that in either case, EF is usually explaining some, but not all, of the variance in the relationship between the precursor skill and the academic outcome.

Rather than pit domain-general and domain-specific capacities against each other, here, we outline a more nuanced framework for relating EF to mathematics. Specifically, we propose that executive functions contribute to general math skills both directly - supporting the execution of problem solving strategies - and indirectly - supporting the acquisition of precursor mathematical content (Bascandziev, Tardiff, Zaitchik, \& Carey, 2018) (Figure 1A), which in turn support broader mathematical learning. Thus, the crucial prediction of this framework is that individual differences in specific precursor mathematical skills should explain (some of) the relationship between specific executive functions and overall math skills. Moreover, we should see these relationships especially in cases where there is a theoretically motivated relationship between the specific executive function, the precursor skill, and the mathematical content domain. In this study, we investigate this hypothesis for the case of inhibitory control and rational number processing.

\subsection{Inhibitory control contributions to math achievement}

Amongst the canonical measures of EF - working memory, the ability to hold and manipulate information in mind; inhibitory control, the capacity to withhold prepotent responses; and cognitive flexibility, the ability to switch between task goals (Diamond, 2013) - recent meta analyses confirm the robust contribution of working memory to math achievement and a smaller but consistent contribution of cognitive flexibility (Friso-van den Bos, van der Ven, Kroesbergen, \& van Luit, 2013; Peng et al., 2016). Less clear is the contribution of inhibitory control to math learning, as findings in this domain are not as robust, especially when taking into account other EF measures (Bull \& Lee, 2014; Lee \& Lee, 2019; Van Dooren \& Inglis, 2015). Moreover, the majority of work on executive function has been done in preschool and primary school aged 


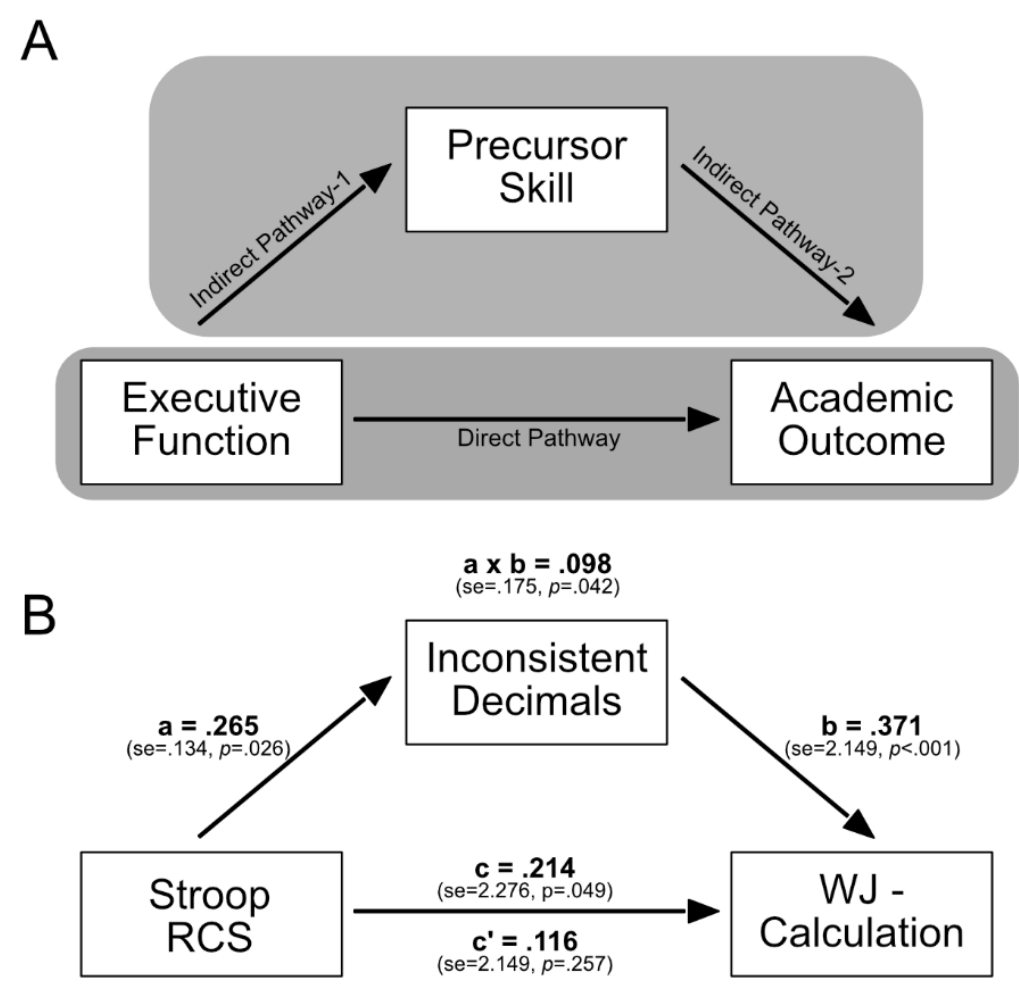

Figure 1. A) Framework for relating executive functions to academic outcomes. The Direct Pathway captures the contribution of a specific executive function on the academic measure. The Indirect Pathways captures the contribution of that executive function in supporting content learning (Indirect Pathway-1), which in turn contributes to performance on the academic measure (Indirect Pathway-2). B) Mediation analysis of the role of inhibitory control on mathematics achievement via decimal processing. The total effect of Stroop Rate Correct Score (RCS, inhibitory control) on Woodcock-Johnson (WJ) - Calculation scores (math achievement) is significant $\left(c=.214^{*}\right)$. Stroop Incongruent also predicts Mixed Inconsistent Decimals performance $\left(a=.265^{*}\right)$, which in turn predicts WJ - Calculation scores $\left(b=.371^{* * *}\right)$. When including the mediating term, the indirect mediation effect is significant $\left(a \times b=.098^{* *}\right)$, while the effect of Stroop RCS on calculation performance is no longer significant $\left(c^{\prime}=.116\right) .{ }^{*} p$ $<.05,{ }^{* *} p<.01,{ }^{* *} p<.001$.

children, with a growing focus on middle and secondary school. Given the ongoing development of executive functions in adolescence and early adulthood (Constantinidis \& Luna, 2019), the first aim of this study was to establish the contributions of inhibition, independent of working memory and cognitive flexibility, to math achievement among a sample of college undergraduates (Figure 1A, Direct Pathway).

\subsection{Role of inhibitory control in rational number understanding}

One possible explanation for the relatively weaker effects for inhibition compared to working memory is that this EF capacity may be particularly important for acquiring and performing mathematical domains that involve acquiring new knowledge that contradicts one's prior knowledge (Wilkinson et al., 2019). Indeed, recent work in science learning reveals a specific role for inhibition in domains that require conceptual change, such as vitalist theories of biology, but not in the rapid acquisition of factual knowledge (Bascandziev et al., 2018). Broad measures 
of mathematics achievement typically mix together these kinds of learning, potentially washing out the effects of inhibitory control. Among mathematical content domains, rational numbers are often cited as a paradigmatic example of conceptual change, requiring expanding one's definition of number beyond the discrete count list view of whole numbers (Carey, 2011; Vamvakoussi \& Vosniadou, 2004), making it an ideal domain to examine the need for inhibitory control in mathematics learning.

Rational numbers are a critical bottleneck in the elementary school curriculum (National Mathematics Advisory Panel, 2008). One proposal for the source of difficulties with rational number learning comes from the fact that properties of rational numbers often contradict learners' prior knowledge of whole numbers (Carey, 2011; Rosenberg-Lee, 2021, March 24). Applying whole number properties when working with rational numbers - termed whole number bias (Ni \& Zhou, 2005) - manifests in children not understanding that, for example, multiplying rationals can result in a smaller number or that there is not a finite set of rational numbers between any two numbers (Kainulainen, McMullen, \& Lehtinen, 2017; Van Hoof, Degrande, Ceulemans, Verschaffel, \& Van Dooren, 2018). The classic demonstration of this phenomenon is in fraction comparison, where individuals are slower and less accurate to select the larger fractions when the correct response is incongruent with whole number knowledge (e.g., 1/2 is larger than 1/9, yet 2 is smaller than 9) (e.g., Bonato, Fabbri, Umiltà, \& Zorzi, 2007). Moreover, negative priming studies demonstrate that solving these counterintuitive problems lowers performance on subsequent consistent problems suggesting participants have inhibited their whole number knowledge, making it less available for consistent problems (Rossi, Vidal, Letang, Houdé, \& Borst, 2019).

Despite general agreement that resolving interference from whole number knowledge is needed to successfully process rational numbers, surprisingly little research has tested this proposal using individual differences designs. In this approach, variation across participants in inhibitory control, measured by a separate task, is related to variation in rational number performance. In children, three studies to date have taken this approach and found moderate evidence to support the predicted relation between inhibition and rational number understanding. Employing the Numerical Stroop task, Gómez, Jiménez, Bobadilla, Reyes, and Dartnell (2015) found that individual differences in inhibition were related to fraction comparison performance, especially on problems incongruent with whole number knowledge. Avgerinou and Tolmie (2019) report that in a Go/No Go task, Go accuracy predicted performance on incongruent fraction and decimal comparison in low load situations, and that Animal Stroop performance scores explained additional variance in cases with higher cognitive load. Finally, Ren and Gunderson (2021) found that inhibitory control, as measured by the Hearts and Flowers task (Wright \& Diamond, 2014), correlated with decimal comparison accuracy in $6^{\text {th }}-8^{\text {th }}$ grade children, during a training session and at immediate and delayed post-tests. By contrast, in adults, Matthews, Lewis, and Hubbard (2016) related nonsymbolic comparison capacities to symbolic fraction and general math skills, while controlling for inhibition. They report that predictions of math outcomes from nonsymbolic comparison performance were robust to the inclusion of a Flanker task. In fact, they found no relationships between Flanker task performance and several measures of overall symbolic fraction understanding.

The tasks used to assess inhibitory control may explain these discrepant findings (Lee \& Lee, 2019). The Flanker task requires reporting the direction of a centrally presented stimulus, which could be flanked by stimuli pointing in the same or opposite directions. Potentially, this type of 
visual distractor inhibition task may not be measuring the relevant aspect of inhibitory control needed for rational number processing (Lee \& Lee, 2019). In contrast, stronger relationships between inhibition and rational number outcomes were found from inhibition tasks which draw on semantic knowledge. Both the Numerical and Animal Stroop tasks require participants to ignore the physical size of the stimuli to select either the larger numerical value or larger animal in real life. Indeed, Stroop-type semantic inhibition tasks have been linked to scientific reasoning, while response inhibition tasks, like Stop-signal reaction time and Go/No Go, have not (Mason \& Zaccoletti, 2020).

Beyond the measure of inhibition, Matthews et al. (2016) differed from Gómez et al. (2015) and Avgerinou and Tolmie (2019) by combining congruent and incongruent problems in one measure of fraction understanding, as well as focusing on adults, rather than children. Constructing fraction comparison stimuli that reliably introduce whole number interference is challenging (Braithwaite \& Siegler, 2018), especially in adults (Obersteiner, Van Dooren, Van Hoof, \& Verschaffel, 2013). Participants use a variety of strategies when comparing fractions, such as comparing numerators only (Obersteiner \& Tumpek, 2016). Participants' application of this strategy can be detected by presenting stimuli where it will lead to correct responses (7/9 vs. $2 / 3$ ) or not (3/5 vs. 4/7). However, one particularly insidious strategy makes this division of stimuli problematic. Common among more sophisticated participants, the gap strategy, involves selecting the fraction with the smaller gap between the numerator and denominator (Obersteiner et al., 2013). In fact, this strategy will always lead to the correct answer on "incongruent" problems (3/5: gap $=2 ; 4 / 7$ : gap $=3)$, while leading participants astray on a subset of "congruent" problems (7/9: gap $=2 ; 2 / 3$ : gap $=1)$ but not others $(3 / 5:$ gap $=2 ; 1 / 4$ : gap $=3)$. We present these details to illustrate that the extent to which a fraction comparison problem draws on inhibition of whole number knowledge will depend on the strategy the participant uses to solve that problem. These factors make fraction comparison a suboptimal choice for assessing the role of inhibitory control on rational number understanding, especially among adults.

Relative to fractions, decimals have received considerably less attention in the research literature. Yet, they share many of the properties of fractions that make rational numbers difficult to master, while circumventing some of the experimental ambiguities introduced by strategy variation in fraction comparison. Among whole numbers, more digits always indicates a larger number, making it harder to determine the larger of 0.9 vs. 0.27 relative to pairs where the larger number also has more digits, such as 0.2 vs. 0.87 . This string length congruity effect (Huber, Klein, Willmes, Nuerk, \& Moeller, 2014) is found in children (Avgerinou \& Tolmie, 2019; Ren \& Gunderson, 2019) and adults (Varma \& Karl, 2013) and even induces priming effects on subsequent processing in children and adolescents (Roell, Viarouge, Houde, \& Borst, 2017, 2019). Other effects have also been investigated in decimal comparison (Huber et al., 2014; Varma \& Karl, 2013), including the unit decade compatibility effect (slower reaction times when the tenths and hundredths digit conflicts, e.g., 0.19 vs. 0.86) and the zero facilitation effect (faster reaction for problems involving zero, see Appendix A). We included stimuli to capture these effects in order to assess the specificity of string length congruity in relating inhibition to math achievement.

In the current study, we used decimal comparison to reliably induce whole number interference in adults (Huber et al., 2014; Varma \& Karl, 2013). On the inhibition side, we employed the Color-Word Stroop task, which requires overcoming learned semantic knowledge, namely the automatic reading of color words. Thus, the second aim of this study was to assess the 
contribution of the executive function capacity of semantic inhibition on the comparison of inconsistent decimal pairs (Figure 1A, Indirect Pathway-1).

\subsection{Rational number understanding as a precursor skill}

The second leg of the indirect pathway from executive functions to mathematics achievement posits a relationship between a precursor skill and a mathematical outcome measure (Figure 1A, Indirect Pathway-2). Building on work identifying symbolic and nonsymbolic whole number comparison and estimations tasks as predictors of math skills (De Smedt et al., 2013; Halberda et al., 2008; Schneider et al., 2017), recent studies have aimed to relate rational number understanding to math outcomes. In terms of symbolic fractions knowledge, Gómez et al. (2015) also report that children who struggled with incongruent fraction comparisons had lower classroom mathematics achievement. Siegler et al. (2012) found that knowledge of fractions in late elementary school predicted high school math achievement in large United States and United Kingdom samples. Interestingly, backward digit span (a measure of working memory capacity) in elementary school also predicted later math outcomes, but when both were included in a regression model, only knowledge of fractions remained a significant predictor. This pattern is consistent with the suggestion that specific math content knowledge can explain the relationships between executive functions and overall math skills.

Another line of research focuses on linking nonsymbolic rational number processing with math achievement. As noted above, Matthews et al. (2016) found that the capacity to compare relative proportions of line lengths or sets of dots predicted greater knowledge of math including performance on a college entrance exam. Similarly, Siegler, Thompson, and Schneider (2011) found that the capacity to accurately estimate the location of fractions on a number line predicted state measures of math achievement over and above the contribution of measures of fraction arithmetic knowledge in $6^{\text {th }}$ and $8^{\text {th }}$ grade children. Accuracy in both fraction and decimal number line placement also correlates with algebra understanding in $7^{\text {th }}$ grade children (DeWolf, Bassok, \& Holyoak, 2015).

These studies suggest that rational number understanding contributes to later high-level math skills. To our knowledge, no research to date has examined relations between decimal comparison tasks and overall math skills in adults. In children, Resnick and colleagues recently found that decimal understanding contributed to both fraction learning and math achievement scores (Resnick, Rinne, Barbieri, \& Jordan, 2019). If processing inconsistent decimals indexes a more general measure of rational number understanding (Avgerinou \& Tolmie, 2019), then we might expect relations to general math skills comparable to those found with fraction tasks. Potentially, the effects could be even stronger for decimals as they are less contaminated by strategy variation than fractions. Alternatively, fractions may be a particularly challenging concept relative to decimals (Kainulainen et al., 2017; Van Hoof et al., 2018), and lack of variation in the capacity among adults may render decimal comparison a weak predictor of math skills. Thus, the third aim of this study was to examine relations between decimal comparison performance (especially of inconsistent problems) and mathematics achievement (Indirect Pathway-2 in Figure 1A).

As previous studies have found that executive functions vary in their contribution depending on the type of math measure (Lee \& Bull, 2016; Peng et al., 2016), here we decomposed our primary outcome measure, the Calculation subtest Woodcock Johnson-III, into two sets of items, rational numbers and advanced concepts not involving rational numbers (i.e., logarithms, 
trigonometry, calculus, etc.). This division enabled us to assess the extent to which variation in decimal performance could explain the contribution of inhibitory control to rational numbers versus other challenging mathematical content. If decimal understanding is a precursor to a broad range of mathematical topics, we would expect it to explain the contribution of inhibition to both sub-measures. Alternatively, it may be a particular stumbling block in rational number learning, and hence explain relatively more of the contribution of inhibition to this domain than advanced concepts such as algebra and trigonometry.

\subsection{The current study}

In the current study, we aimed to relate executive function measures to math achievement via a precursor skill. We employed tablet-based rapid assessments of EF capacities (Younger et al., 2021, April 20). Working memory and inhibition were assessed using computerized versions of classic tasks in the field: backward Corsi Block Tapping to measure visual spatial working memory and Color-Word Stroop to measure inhibitory control. Cognitive flexibility was measured using TaskSwitch, a task where participants switched between applying rules to evaluate a centrally presented visual stimuli. Our target precursor skill was a decimal comparison task where correct responses were inconsistent with prior whole number knowledge (e.g., 0.27 vs. 0.9). In terms of mathematics achievement, our primary outcome measure was the Calculation subtest of the Woodcock Johnson; we also collected Math Fluency to account for lower level math knowledge. Also, relations between EF factors and mathematical outcomes likely vary depending on the type of mathematics assessed (Lee \& Bull, 2016; Peng et al., 2016). Accordingly, we decomposed our primary untimed measure of mathematics knowledge into a rational number and advanced math sub-scores.

We first sought to establish that inhibitory control is an independent predictor of math achievement in young adults (Direct Pathway). Next, we hypothesized that individual differences in inhibitory control would predict inconsistent decimal comparison performance (Indirect Pathway-1), which in turn would predict mathematical outcomes (Indirect Pathway-2). This pattern of results would lend support to our framework, emphasizing that the contributions of executive functions to mathematical outcomes can be explained in part by their role in the acquisition of precursor skills which support advanced mathematical knowledge. Further, we examined whether inconsistent decimal comparison performance explains more of the relationship of inhibition with rational numbers than with advanced concepts.

\section{Methods}

\subsection{Participants}

Participants were Rutgers University-Newark undergraduate students taking psychology courses who received course credit for participation. Among the 114 individuals that participated in the study, technical issues resulted in data loss for 8 participants ( 2 missing math measures, 1 missing the Decimals task, and 5 missing one or more executive function tasks). Only participants with full data sets were included. Further, following Gómez et al. (2015), we only considered data from participants who performed above $30 \%$ in overall accuracy on the Decimals task ( $n=9$ failed to meet this criterion). 
The final sample consisted of 97 participants (Mean age $=20.58$ years, $S D=1.79$, Table 1 ). Participants were asked both their self-identified gender and their sex assigned at birth. For gender, 79 participants identified as female and 18 as male. Amongst the female self-identified, four declined to provide a sex at birth, while among self-identified males one participant gave female as their sex at birth. In all other cases, sex assigned at birth and gender selfidentification corresponded (95\% of participants). For race, $28 \%$ were Black/African American, 21\% were Hispanic/Latino, 19\% were Asian/Pacific Islander, 11\% Middle Eastern/Arab/Persian and $11 \%$ were White/European. The remaining participants identified as other $(7 \%)$ or declined to answer (3\%). In terms of academic major, science majors were most common including psychology (25\%), biology (20\%), computer science $(4 \%)$ and $1-2 \%$ of students in neuroscience, engineering or nursing. Among the remaining participants with declared majors, area of study included social sciences $(8 \%)$, criminal justice $(7 \%)$, humanities (5\%), social work $(5 \%)$, business $(3 \%)$ and fine arts (1\%). Finally, $10 \%$ of participants had yet to declare a major and $6 \%$ declined to answer.

Based on prior work suggesting common effect sizes in correlational studies of mathematical precursor skills of $r=.30$ (Schneider et al., 2017), we determined that a sample sizes of 84 participants would be sufficient to detect these effects with $80 \%$ power and alpha $=.05$. Given prior recruitment rates and the uncertainties of data loss, we reasoned that two semesters of data collection (Fall 2017, Spring 2018) through the departmental participant pool would ensure a sample exceeding that size. Informed consent was obtained prior to data collection and the study was approved by the Rutgers University Institutional Review Board.

\subsection{Decimals Comparison Task}

Stimuli, conditions, and task timing were modeled after Experiment 3 of Varma and Karl (2013), except participants did not receive feedback on their performance and some of the stimuli were modified to better balance across conditions (see Appendix A for the full stimuli set). The task was implemented in PsychoPy 1.85 .2 (Peirce, 2007), on a 14 inch Lenovo ThinkPad laptop and began with a practice block consisting of two trials of whole number comparison followed by four trials of one-digit decimal comparison. Each trial began with a 500 ms fixation screen, followed by presentation of the decimal pairs for $3000 \mathrm{~ms}$, followed by a blank screen for $500 \mathrm{~ms}$.

Participants were instructed to indicate which decimal was greater by pressing either the " $z$ " key if the larger quantity was on the left or the "m" key if it was on the right (corresponding to the spatial locations of the decimals on the screen). See Figure 2 for an example of stimulus presentation. All text was rendered in Arial font in white characters with a black background. The decimals stimuli were displayed with letter height of .15 (normalized units in PsychoPy), which resulted in numbers of height $1 \mathrm{~cm}$ on the laptop.

All decimal stimuli began with "0.", but differed in number and type of digits following the decimal. 1) Uniform stimuli consisted of pairs where both decimals had 2 digits after the decimal point. 2) Mixed stimuli comprised of one decimal with one digit after the decimal point and the other had 2 digits. Finally, 3) Zero stimuli were constructed by adding a "0" after the single-digit decimal in the mixed condition. Consistency was also manipulated (consistent and inconsistent) but indexed different effects depending on condition. For Uniform trials, the tenths and hundredths values were manipulated so that either comparing both numbers led to the same conclusion (consistent: 0.21 vs. 0.87 ) or not (inconsistent: 0.29 vs. 0.83 ), an effect termed the unit-decade compatibility effect in whole numbers (Nuerk, Moeller, Klein, Willmes, \& Fischer, 2011; Nuerk, Weger, \& Willmes, 2001). For Mixed stimuli, both the number of digits and the 
numerical quantity led either to the same conclusion (consistent: 0.2 vs. 0.87 ) or not (inconsistent: 0.27 vs. 0.9). For the Zero stimuli, adding the zero removes the inconsistency between number of digits and magnitude; however, we still use the terminology of consistent ( 0.20 vs. 0.87$)$ and inconsistent $(0.27$ vs. 0.90$)$ to align with the corresponding Mixed conditions (Appendix A). Including Zero stimuli enabled us to confirm that poorer performance in the Mixed Inconsistent condition was due to interference from the number of digits, not the specific numerical values employed. There were 14 unique pairs in each of the six conditions and each were presented twice - once with the larger stimuli on the right and once with the larger on the left - for a total of 168 trials. Presentation of decimal pairs were counterbalanced across two blocks, with a self-paced break between them, which also reminded participants of the task instructions. We excluded anticipatory responses (reaction times shorter than $250 \mathrm{~ms}$ ) and outlier responses (reaction times $3 \mathrm{SD}$ above an individual's mean). After applying these criteria, we analyzed 15874 (97.9\%) out of a total of 16212 trials. Due to experimenter error one participant had only one block (84 trials) on the Decimals Comparison Task, but otherwise a full data set, and so was included in all analyses. We computed accuracy and reaction times for the six conditions, and our primary measure of interest was accuracy on the Mixed Inconsistent condition.

\subsection{Standardized Measures of Mathematical Achievement}

Participants were administered the Woodcock Johnson III Test of Achievement (Woodcock, McGrew, \& Mather, 2001) subtests of Calculation and Math Fluency, from Version A.

Calculation measures a person's ability to complete mathematical problems that increase in difficulty starting from basic arithmetic up to integral calculus. Participants were given as much time as they needed to complete this subtest. To compute the Rational Number sub-score we summed each individual's scores on the seven rational number items in the Calculation subtest involving arithmetic of proper fractions (items: $20,23,30)$, mixed fractions $(25,27,36)$, and computing portion from a percentage (34). To avoid circularity with the decimal comparison task, we did not include the decimal multiplication item (26) in the Rational Number sub-score. Ten items made up the Advanced Math sub-score in the domains of cube roots (31), systems of linear equations $(32,42)$, quadratic equations (33), logarithms (37), calculus $(40,44)$, trigonometry $(41,45)$, and matrix determinants (43). We omitted three items with exponents and square roots which also involved fractions $(35,38)$ or decimals $(39)$. Finally, no whole number $(1-19,21,22,24)$ and integer arithmetic items $(28,29)$ were included in either measure.

Math Fluency assesses a person's ability to rapidly complete up to 160 single-digit vertically presented addition, subtraction, and multiplication problems within a three-minute time limit. We employed the Math Fluency subtest to control for general whole number arithmetic understanding in the secondary regression analyses assessing the contribution of decimal understanding to explaining relations between inhibition and specific mathematical domains.

\subsection{Executive Function Tasks}

The Adaptive Cognitive Evaluation (ACE) program is a recently developed tablet application for the iPad with a variety of games aimed at measuring different aspects of executive function (Younger et al., 2021, April 20). Among the nine experimental tasks, we selected three games: Color-Word Stroop to measure inhibitory control, TaskSwitch to measure cognitive flexibility, and Backward Spatial Span to measure visual spatial working memory. Prior to completing the executive function tasks, participants first completed a computerized version of the Ishihara 
colorblindness test within ACE (Ishihara, 1972), as two of the tasks required the ability to differentiate between green and red. This task consisted of 2 trials where participants had to identify a numeral presented in green circles in an array of red circles. All participants passed this test. Participants next completed a basic reaction time assessment to determine how quickly they could make screen taps with their left and right hands. Due to technical issues, data for left hand responses was lost from 14 participants, and among these 5 were also missing right hand responses. For the 83 participants with data for both sides, reaction times were faster for the right than the left side $(280 \mathrm{~ms}$ vs. $305 \mathrm{~ms}, t(82)=6.43, p<.001)$.

The ACE suite of games (Younger et al., 2021, April 20) provides participants with trial level feedback in all tasks. After each trial, a green, yellow, or red centralized fixation cross was displayed for $200 \mathrm{~ms}$ (500ms in the case of Backward Spatial Span). Green indicated a correct response within the time interval, yellow a correct response beyond the time window, and red an incorrect response. For Stroop and TaskSwitch, each trial was followed by $1000 \mathrm{~ms}$ inter trial interval, whereas for Backwards Spatial Span, the interval was $500 \mathrm{~ms}$. One challenge of employing robust cognitive tasks in individual difference designs is the low reliability of difference/cost scores within individuals, despite the consistency of cost effects across the population (Hedge, Powell, \& Sumner, 2018). Notably, for inhibition and cognitive flexibility tasks, accuracy and reaction time within conditions tend to have higher interclass correlation (a measure of reliability) compared to cost measures (Hedge et al., 2018). Thus, we employed rate correct score (RCS) (Vandierendonck, 2017) of the full task in the regression analyses. We reasoned that this metric offered the best method of capturing individual variation in executive function tasks, to help address our primary question regarding understanding covariance relations between executive functions and math achievement.

2.4.1. Stroop. In this modification of the Stroop (Stroop, 1992) paradigm for manual responses (Mead et al., 2002) in a mobile computing environment, participants were presented with four response options - red, green, yellow, and white circles - which were displayed at the bottom of the screen (see Figure 2). They were instructed to place index and middle fingers over the circles and to respond with the ink color of the presented word. In congruent trials, a color word (e.g., "GREEN") would be displayed in the same color ink (e.g., green). Incongruent trials involved presenting the color word in a different color ink (e.g., red). Participants completed two rounds of practice. The first had 15 trials of simply selecting the color of a circle based on colored "XXXX" and the second had 10 interspersed congruent and incongruent trials. The task consisted of 50 trials, 35 congruent and 15 incongruent. Participants were required to respond during the stimulus display, which was initialized at $800 \mathrm{~ms}$ and adapted thereafter depending on participant performance. Following the same procedure as in the Decimal Comparison Task to exclude anticipatory and outlier responses, we analyzed 4818 (99.34\%) out of a total of 4850 trials. Due to the imbalance of problems in each condition and the adaptive nature of the presentation time, we computed rate correct score (RCS) to summarize participant performance across the entire task. This metric computes the total number of correct responses divided by the total reaction time across correct and incorrect trials (Vandierendonck 2017). RCS can be thought of as the number of correct responses per unit time.

2.4.2. TaskSwitch. This task assesses participants' ability to quickly shift between one set of task rules. They were given four response choices at the bottom of the screen, a triangle (shape response), a square (shape response), a green circle (color response), and a red circle (color response). Participants were presented with the cue "shape" or "color" and were next presented 
with a target-a shape (triangle or square) in a particular color (red or green) during the test probe (Figure 2). Participants were instructed to select either the color or the shape of the stimulus, depending on the cue. The cue and delay period were presented for random intervals ranging from $800-1200 \mathrm{~ms}$ in $100 \mathrm{~ms}$ increments. Participants were required to respond during the test probe, which was initialized at $800 \mathrm{~ms}$, and adapted thereafter depending on their performance. Stay trials were those that presented the same cue (either "shape" or "color") for two consecutive trials. Switch trials were those where the cue on the previous trial was different from the current trial (e.g., first "shape" and then "color", or vice versa). Participants completed 10 practice trials before the start of the task which consisted of 30 trials. After discarding the first trial, which is neither a stay nor switch trial, there were 10 switch and 19 stay trials. Following the same procedure as in the Decimal Comparison Task, to exclude anticipatory and outlier responses, we analyzed $2869(98.59 \%)$ out of a total of 2910 trials. We used RCS as the primary metric to summarize TaskSwitch performance.

2.4.3. Backward Spatial Span. Participants' visual spatial working memory was assessed using a tablet version of the Corsi Block Tapping task (Corsi, 1972). Participants were presented with a screen of 20 small circles, which briefly lit up in a variable sequence (Figure 2). Participants were prompted to tap the sequence of illuminated circles in reverse order. After completing two practice rounds of 3 locations, participants proceeded to the main task, where the length of the sequences increased adaptively from 3 to 8 locations. At each level, participants had up to 3 opportunities to successfully produce 2 sequences before moving to the next level. If participants could not do so, the task ended. If participants could not produce any correct sequences at 3 locations, they were assigned a span of 2 . Typically in Corsi tasks, the longest sequence successfully produced a certain number of times is considered that participant's object span. In order to capture participants' variability in this task for correlational analyses, we modified this scoring procedure to award participants an extra half point if they were able to successfully produce 1 sequence at the length beyond their complete spatial span. We term this value Spatial Span and used it as the primary metric to summarize backward spatial span.
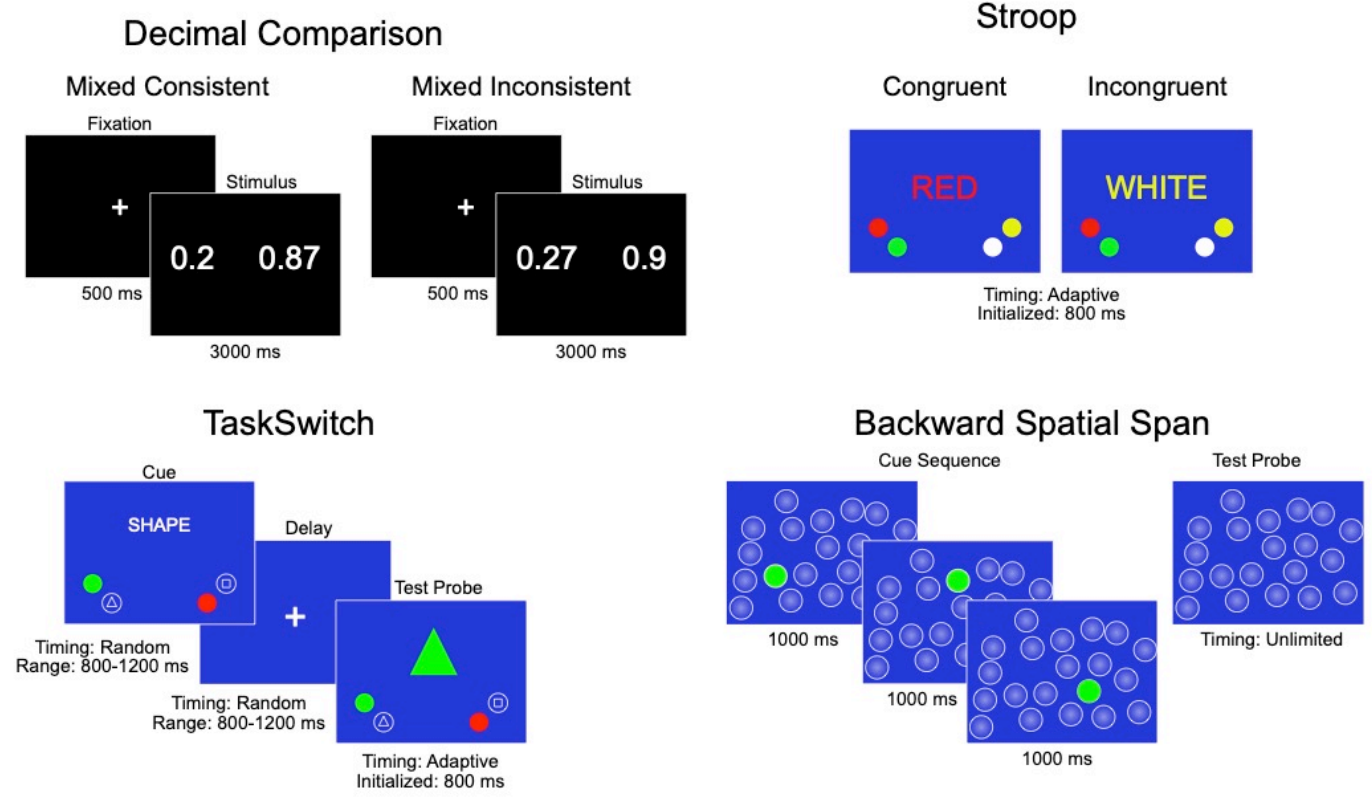
Figure 2: Schematic representations of experimental tasks. Decimal Comparison task had a fixed timing structure of 500 ms Fixation cross followed by a 3000 ms Stimulus presentation. Mixed stimuli compared 1 digit decimals with 2 digit decimals which could be Consistent or Inconsistent with whole number knowledge (not shown: Zero and Uniform conditions, see Appendix A). Participants indicated the larger numerical quantity with a left or right keyboard press. Stroop task stimuli were initially presented for $800 \mathrm{~ms}$, but subsequent timing adapted depending on participants' performance. Participants viewed a centrally presented color word whose ink color could be Congruent or Incongruent, and indicated the font color, via touch screen, of the corresponding colored circle. TaskSwitch task began with a Cue (800-1200 ms) alerting participants to the feature they should report in the test probe, followed by a Delay period with fixation cross (800-1200 ms). In the Test Probe, a centrally presented shape, either a triangle or square, was presented in either green or red. Participants indicated the corresponding value for the cued feature on the touch screen. In this example, the trial began with the cue "shape", followed by the target of a green triangle. The correct response in this case would be the triangle. Presentation time of the text probe was initially $800 \mathrm{~ms}$, but subsequent timing adapted depending on participants performance. Stay trials were those that presented the same cue (either "shape" or "color") for two consecutive trials. Switch trials were those where the cue on the previous trial was different from the current trial (e.g., first "shape" and then "color" or vice versa). Backward Spatial Span presented sequences of illuminated circles (Cue Sequence) for 1000 ms each. During the Test Probe, participants had to recreate the sequence in reverse presentation order on the touch screen. Participants had unlimited time to make their responses.

\subsection{Experimental Procedure}

The experimental session consisted of the decimals comparison task, completed on a ThinkPad laptop (Lenovo, Hong Kong), followed by the two paper measures of math achievement from the Woodcock Johnson III Tests of Achievement (WJ-III). Participants next completed the ACE tablet assessments of EF on a 9.7 inch iPad 2017 (Apple, Cupertino, CA). Finally, using Qualtrics (Provo, UT) accessed through the tablet's browser, participants completed a demographics questionnaire.

\subsection{Analyses}

Analyses were conducted in R (R Core Team, 2018) and Jamovi (The jamovi project, 2020). For regression analyses, all mentions of variance explained use adjusted- $R^{2}$, and "additional variance" refers to the difference between adjusted- $R^{2}$ for nested regression models. To compute the significance of direct and indirect effects in the mediation analyses, we modified the Sobel function of the $\mathrm{R}$ library multilevel (Bliese) to allow for multiple control regressors. To compute percent mediation, we used the formula (Beta Direct $_{-}-$Beta $_{\text {Indirect }}$ )/Beta Direct $_{\text {(Kenny, }}$ Korchmaros, \& Bolger, 2003).

\section{Results}

\subsection{Standardized math performance}

Aged-normed standardized scores indicate that participants generally performed in the average range on Math Fluency $(M=93.51, S D=13.77)$ and Calculation $(M=101.84, S D=13.09)$. Due 
to the negative correlations between age and standardized math scores (Math fluency: $r(95)=$ $.23, p=.025$; Calculation: $r(95)=-.48, p<.001)$, we used raw scores, along with age, in subsequent regression analyses (Table 1).

Table 1: Average performance on standardized math measures (raw scores) and experimental task (percent correct, or modified spatial span)

\begin{tabular}{rccc}
\hline Measure & $\mathrm{M}$ & SD & Range \\
\hline Age (years) & 20.58 & 1.79 & $18.0-24.8$ \\
WJ - Math Fluency & 110.40 & 23.71 & $50-160$ \\
WJ - Calculation & 29.11 & 5.28 & $14-43$ \\
Decimal Comparison & $91.9 \%$ & $8.7 \%$ & $58.5-99.4 \%$ \\
Stroop & $85.5 \%$ & $9.4 \%$ & $65.3-100 \%$ \\
TaskSwitch & $72.8 \%$ & $2.4 \%$ & $10.0-100 \%$ \\
Spatial Span & 4.52 & 1.27 & $2.0-7.0$ \\
\hline
\end{tabular}

Note: Decimals, Stroop \& TaskSwitch values represent average percent correct in each task. Spatial Span value is average modified location span. WJ = Woodcock-Johnson.

\subsection{Decimals Comparison}

In the Decimals Comparison Task, accuracy was lowest on the critical Mixed Inconsistent trials $(77.6 \%)$, where the correct rational number response conflicted with whole number knowledge, and quite high for all the other conditions (94.1 - 95.7\%, Figure 3, Table 2). A 2-way repeated measures ANOVA confirmed a significant interaction $\left(F(2,192)=31.66, p<.001\right.$, partial $\eta^{2}=$ .248) between Format (Mixed, Zero, Uniform) and Type (Consistent, Inconsistent). There were also main effects of Format $\left(F(2,192)=39.75, p<.001\right.$, partial $\left.\eta^{2}=.293\right)$ and Type $(F(1,96)=$ 18.04, $p<.001$, partial $\eta^{2}=.158$ ). Post-hoc Bonferroni-corrected $t$-tests confirmed that Mixed Inconsistent trials were significantly less accurate than each of the five other conditions (all $p s<$ .001 ), and that none of these conditions differed from each other (all $p s=1.000$ ). The effect of the string length congruity effect on accuracy was shown in an F-test comparing Mixed Consistent and Inconsistent comparisons $\left(F(1,96)=29.23, p<.001\right.$, partial $\left.\eta^{2}=.233\right)$.

Table 2: Means and standard deviations for all the conditions in the Decimals comparison task.

\begin{tabular}{lcccccc}
\hline & \multicolumn{2}{c}{ Mixed } & \multicolumn{2}{c}{ Zero } & \multicolumn{2}{c}{ Uniform } \\
& Consistent & Inconsistent & Consistent & Inconsistent & Consistent & Inconsistent \\
\cline { 2 - 6 } $\begin{array}{l}\text { Accuracy } \\
\text { (percent correct) }\end{array}$ & $94.6(12.4)$ & $77.6(28.5)$ & $94.1(10.7)$ & $95.0(9.4)$ & $95.7(6.7)$ & $94.4(7.2)$ \\
$\begin{array}{l}\text { Reaction time } \\
(\mathrm{ms})\end{array}$ & $923(179)$ & $1048(214)^{1}$ & $935(160)$ & $955(151)$ & $960(161)$ & $948(163)$ \\
\hline
\end{tabular}

${ }^{1} 5$ participants had zero accuracy in this condition, resulting in $\mathrm{n}=92$.

This pattern of results was mirrored in the reaction time (RT) data where Mixed Inconsistent was the slowest condition (1048 ms); the other conditions each averaged less than a second (923$962 \mathrm{~ms}$, Figure 3, Table 2). (The five participants with 0\% accuracy in the Mixed Inconsistent condition were excluded from the RT analyses.) A 2-way repeated measures ANOVA confirmed a significant Format by Type interaction $\left(F(2,182)=28.33, p<.001\right.$, partial $\left.\eta^{2}=.237\right)$. There 
were also main effects of Format $\left(F(2,182)=17.24, p<.001\right.$, partial $\left.\eta^{2}=.159\right)$ and Type $(F(1$, $91)=28.06, p<.001$, partial $\eta^{2}=.236$ ). Post-hoc Bonferroni-corrected $t$-tests confirmed that Mixed Inconsistent trials were significantly slower than all the other conditions (all $p s<.001$ ) and no other conditions differed from each other (all $p s>.18$ ). Again, the effect of the string length congruity effect on RTs was corroborated in an F-test comparing Mixed Consistent and Inconsistent comparisons $\left(F(1,91)=37.78, p<.001\right.$, partial $\left.\eta^{2}=.293\right)$. Together with the accuracy findings, these results demonstrate the robustness of interference from whole number knowledge when comparing decimals quantities.
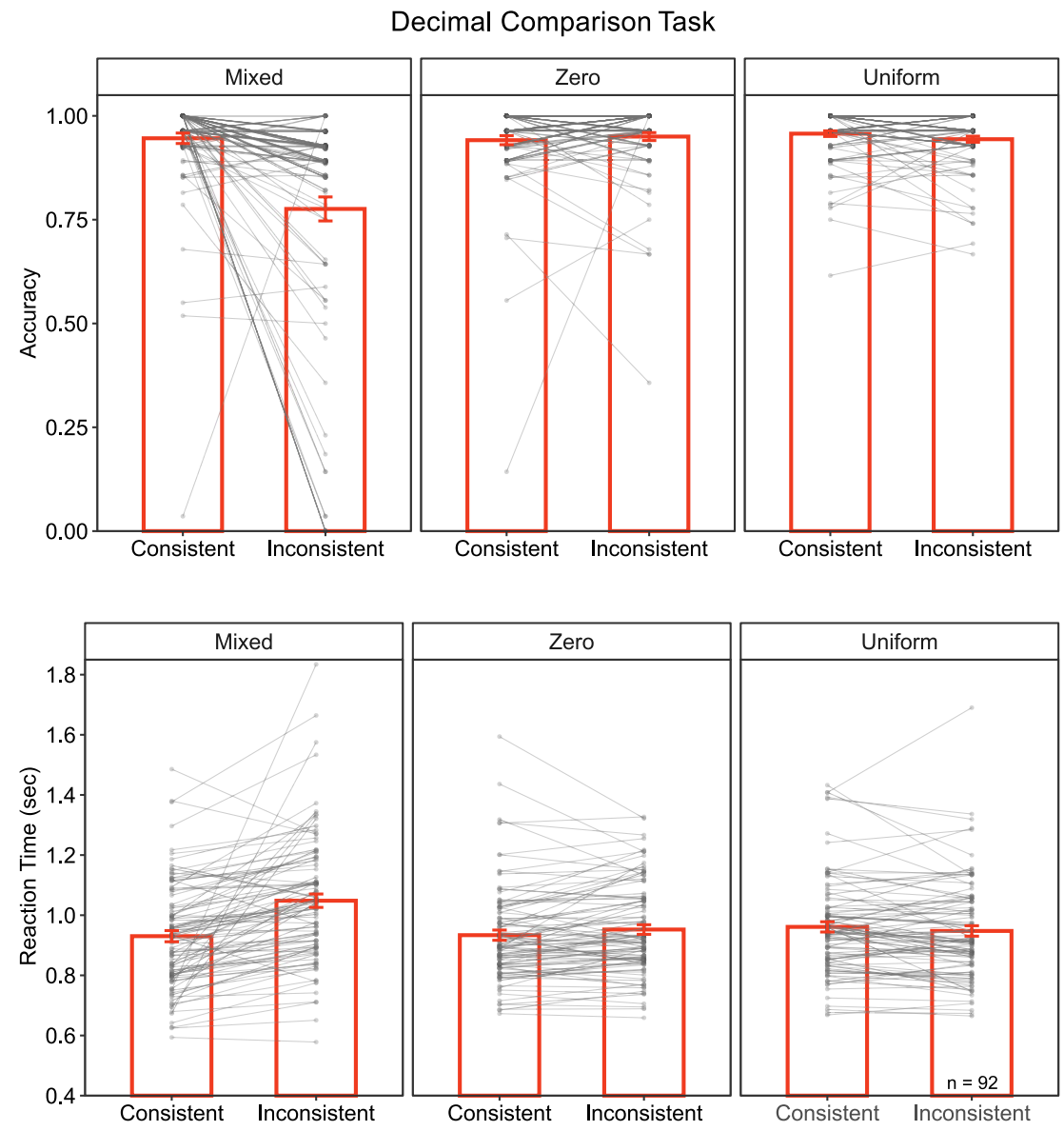

Figure 3: Decimals Task performance. Participants were less accurate $(p<.001)$ and slower $(p<.001)$ in Mixed Inconsistent $(0.27$ vs. 0.9$)$ than Mixed Consistent $(0.2$ vs. 0.87$)$. There were no differences in accuracy $(p s=1.00)$ or reaction time $(p s>.18)$ between Zero Consistent $(0.20$ vs. 0.87 ) or Inconsistent (0.90 vs. 0.27 ), nor between Uniform Consistent (0.21 vs. 0.87 ) and Inconsistent ( 0.29 vs. 0.83 ). Note: 5 participants had $0 \%$ in the Mixed Inconsistent condition, thus Reaction time data only presents participants with full data sets $(n=92)$.

\subsection{Inhibitory control}

As expected participants had lower accuracy on Stroop Incongruent (63.0\%) than Congruent trials $(95.0 \%)$, a significant difference $(t(96)=10.60, p<.001$, Cohen's $d=1.08$, Figure 4). Moreover, when participants were able to respond correctly, they had slower RTs on the Incongruent $(676 \mathrm{~ms})$ than Congruent $(616 \mathrm{~ms})$ trials $(t(90)=-7.83, p<.001$, Cohen's $d=-0.82$, 
Figure 4). Note that 6 participants were excluded from RT analysis due to $0 \%$ accuracy in the Stroop Incongruent condition. We used the Rate Correct Score (RCS) measure for subsequent correlation and regression analyses to combine both accuracy and reaction time over the entire task $(M=1.36, S D=.25$, Figure 5$)$.

\subsection{Cognitive flexibility}

In the TaskSwitch task, accuracy was fairly low for both Stay $(73.6 \%)$ and Switch $(73.0 \%)$ trials and there was no significant difference between the conditions $(t(96)=-.36, p=.718$, Cohen's $d$ $=0.04$, Figure 4). Average accuracy on the first trial, which could not be considered either a Stay or Switch trial, was $54.6 \%$. In RT, Stay (663 ms) trials were actually slightly slower than Switch (625 ms) trials, but this difference was not significant $(t(95)=-1.04, p=.301$, Cohen's $d$ $=0.11$, Figure 3). Note that one participant was excluded from the RT analyses for $0 \%$ accuracy on the Switch trials. As can be seen in Figure 4, several participants had very long average RT in the TaskSwitch Task. To confirm that these participants were not contributing to the null reaction time results, we removed participants with average RT on either Stay or Switch trials greater than $1758 \mathrm{~ms}$, which corresponds to the mean RT in the task ( $M=648 \mathrm{~ms}$ ) plus 2 standard deviations ( $S D=555 \mathrm{~ms}$ ). This criterion removed an additional 4 participants, but the effect of trial type remained insignificant $(t(91)=0.66, p=.513$, Cohen's $d=0.07)$. As in Stroop, we used the RCS measure to summarize TaskSwitch performance $(M=1.36, S D=.25$, Figure 5) for correlation and regression analyses.
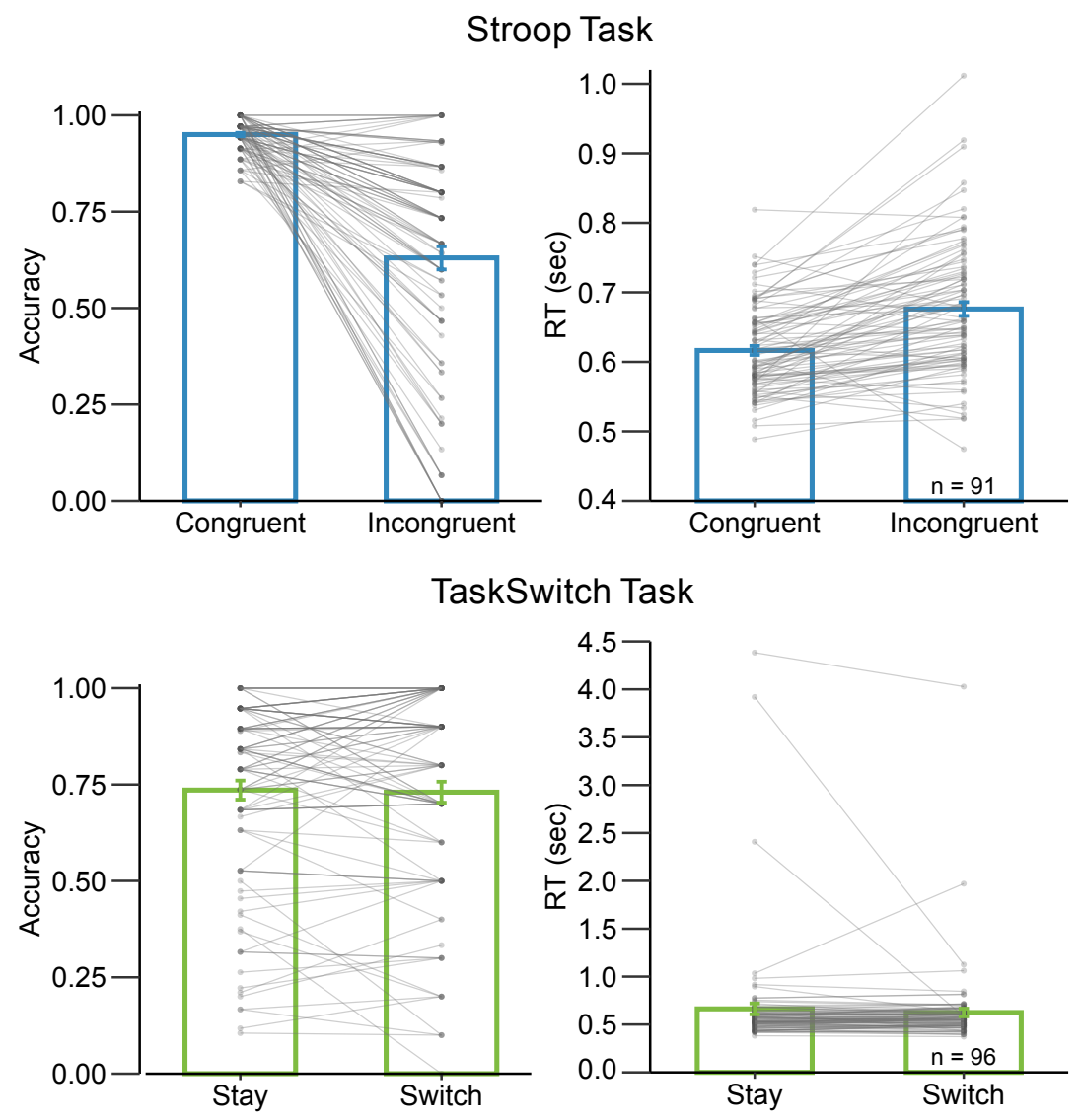
Figure 4: Accuracy and reaction time on Stroop and TaskSwitch. Stroop: Participants were slower $(p<.001)$ and less accurate $(p<.001)$ on Stroop Incongruent than Congruent.

TaskSwitch: There were no differences between Stay and Switch trials in the TaskSwitch Task for accuracy $(p=.718)$ or reaction time $(\mathrm{RT}, p=.301)$. Note: $\mathrm{p}$-values from paired t-tests.

\subsection{Visual-spatial working memory}

Performance on the Backward Spatial Span task ranged from 2 to 7 with average span length of 4.52. See Figure 5 for distribution of Spatial Span scores and Table 1 for the summary statistics.
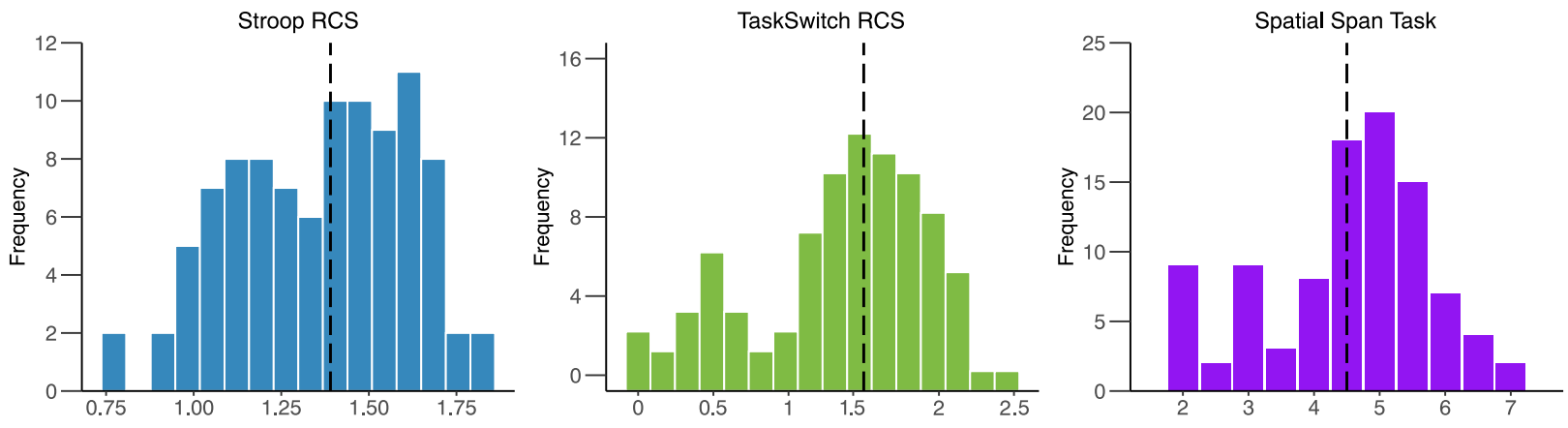

Figure 5: Distribution of performance on executive function tasks. Histograms of the Rate Correct Score (RCS) for Stroop (the measure of inhibitory control), TaskSwitch (the measure of cognitive flexibility), and Spatial Span (the measure of working memory). Dashed lines represent the means.

\subsection{Correlations between measures}

We examined correlations between raw scores on the two mathematics measures, the three executive function tasks, (i.e., Stroop RCS, TaskSwitch RCS, Spatial Span) and the Decimal Comparison Task (i.e., Mixed Inconsistent Decimals) (Table 3). Rate correct score (RCS), which indexes the number of correct responses per unit of task time, was used to summarize performance on the Stroop and TaskSwitch tasks, to account for the adaptivity and imbalanced condition counts in these tasks. Although the raw scores of the two standardized math measures were moderately significantly correlated with each other $(r(95)=.45, p<.001)$, they differed in their relations with the executive function measures. For Math Fluency, there were no significant correlations with any of the EF measures (all ps > .13). By contrast, each of the EF measures correlated with Calculation: Stroop RCS $(r(95)=.28, p=.006$, Figure 6), TaskSwitch $\operatorname{RCS}(r(95)=.21, p=.037)$, and Spatial Span $(r(95)=.25, p=.012)$.

Table 3: Correlations between age, standardized math measures, key executive function and decimal conditions.

\section{Age \\ Fluency Calculation Stroop TaskSwitch Span}

Math Fluency -.128

Calculation $-.445^{\star * *} \quad .452^{* * *}$ 


\begin{tabular}{|c|c|c|c|c|c|c|}
\hline Stroop RCS & -.046 & .154 & $.280^{* *}$ & & & \\
\hline TaskSwitch RCS & $-.224^{*}$ & .038 & $.212^{*}$ & $.507^{*}$ & & \\
\hline Spatial Span & -.014 & .105 & $.253^{*}$ & $.465^{* *}$ & $.441^{* * *}$ & \\
\hline $\begin{array}{r}\text { Mixed Inconsistent } \\
\text { Decimals }\end{array}$ & -.109 & $.362^{\star \star *}$ & $.474^{\star * *}$ & $.294^{* *}$ & .089 & $.297^{* *}$ \\
\hline
\end{tabular}

Next, we considered correlations with performance on the Decimal Comparison Task, specifically the Mixed Inconsistent trials. Here, we found a significant relationship with both Calculation $(r(95)=.47, p<.001$, Figure 6$)$ and Fluency $(r(95)=.36, p<.001)$ performance. In terms of executive functions, performance on this task was also correlated with Stroop RCS $(r(95)=.29, p=.003$, Figure 6) and Spatial Span $(r(95)=.30, p=.003)$, but not with TaskSwitch RCS $(r(95)=.09, p=.384)$. For completeness, Supplementary Table S1 lists the correlations of all six decimal task conditions with Calculation and with the EF tasks. Mixed Inconsistent comparison was the only condition to correlate significantly with Stroop RCS (all other $r s<.18$ ), and it had the highest correlation with Calculation (all other $r s<.28$ ). We also examined correlations of Calculation and the EF tasks with performance on the basic reaction time task among the participants for whom we had data. With the exception of a significant correlation of TaskSwitch RCS with left hand responses $(r(81)=-.23, p=.040)$ and a marginal relation with right hand responses $(r(90)=-.19, p=.078)$, there were no other significant correlations with the basic reaction time tasks (all $p s>.65$, Supplementary Table $S 1$ ).
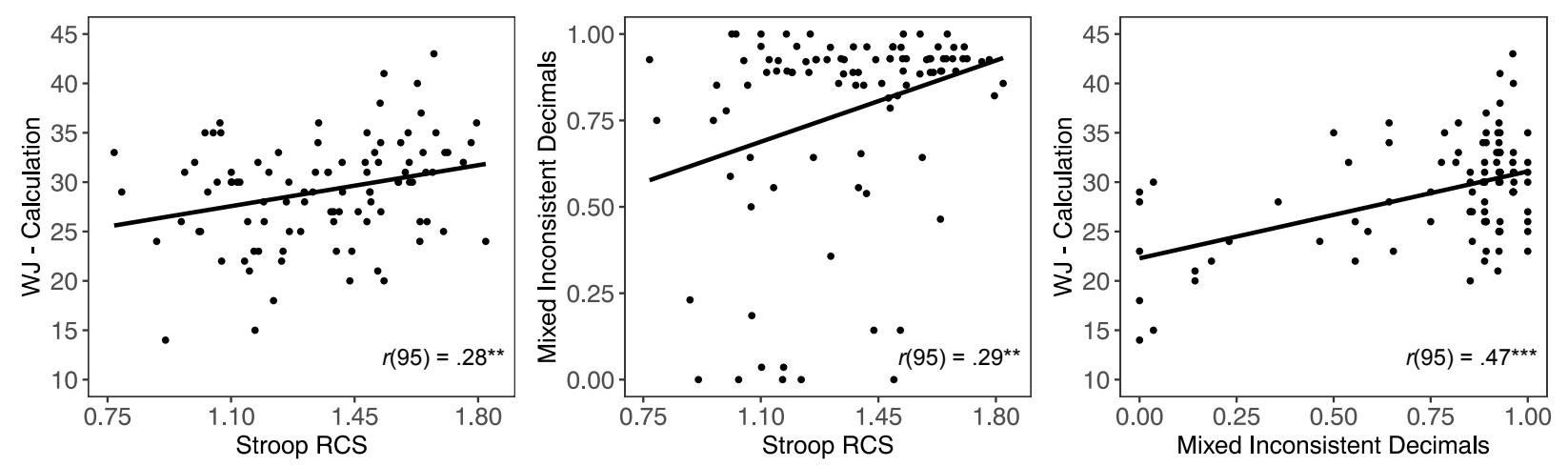

Figure 6: Correlations between nodes in pathway model. Accuracy on the Stroop RCS trials correlated with Woodcock-Johnson (WJ) - Calculation scores and with accuracy on the Mixed Inconsistent trials of the Decimal comparison task. Mixed Inconsistent Decimal performance also correlated with WJ - Calculation scores. ${ }^{*} p<.05,{ }^{* *} p<.01,{ }^{* *} p<.001$.

\subsection{Regression analyses - controlling for executive functioning}

First, we sought to establish that inhibitory control has an independent contribution to inconsistent decimal comparison, over and above the other canonical executive functions. A model with just measures of working memory and cognitive flexibility was significant $(F(2,93)=$ 
3.627, $p=.016$, Table 4, Model A) and explained $7.6 \%$ of the variance in Mixed Inconsistent Decimal accuracy, although only Spatial Span was a significant predictor $(p=.003)$. Adding in inhibitory control to the model fit the data significantly better $\left(F_{\text {change }}(1,92)=5.089, p=.026\right.$, Table 4, Model B), and explained an additional 3.9\% of the variance. Both Spatial Span ( $p=$ .027 ) and Stroop RCS ( $p=.026$ ) were significant predictors. (Although age did not predict decimals performance, we included it in this model to facilitate later computations of mediation effects.)

Table 4: Hierarchical regression analysis of Mixed Inconsistent Decimal comparison performance. (A) Contributions of executive function measures of visuo-spatial working memory (Spatial Span) and cognitive flexibility (TaskSwitch RCS). (B) Addition of inhibitory control (Stroop RCS) to model significantly improved the fit.

\begin{tabular}{llllll}
\hline \multicolumn{7}{l}{ Mixed Inconsistent Decimal Accuracy } & & & \\
\hline Model A & B & SE & Beta & t & p \\
\hline Intercept & .898 & .354 & & 2.540 & .013 \\
Age & -.020 & .016 & -.124 & -1.226 & .223 \\
TaskSwitch RCS & -.041 & .054 & -.085 & -.758 & .450 \\
Spatial Span & .075 & .025 & .333 & 3.030 & .003 \\
\hline Model B & & & & & \\
\hline Intercept & .671 & .360 & & 1.861 & .066 \\
Age & -.022 & .016 & -.136 & -1.370 & .174 \\
TaskSwitch RCS & -.091 & .058 & -.188 & -1.575 & .119 \\
Spatial Span & .057 & .025 & .255 & $\mathbf{2 . 2 5 4}$ & .027 \\
Stroop RCS & .302 & .134 & .265 & $\mathbf{2 . 2 5 6}$ & .026 \\
\hline RCS = rate correct score
\end{tabular}

RCS = rate correct score.

Second, we examined the independent contributions of each executive function measure on math achievement, as measured by the WJ-III subtest Calculation. We included age here due its negative relationship with Calculation raw scores (Table 2). This model was significant $(F(4$, $92)=9.391, p<.001$, Table 5 , Model A) and explained $25.9 \%$ of the variance in scores. Inhibitory control, as measured by performance on the Stroop RCS, was the only EF measure to independently predict Calculation scores $(p=.049)$, although Spatial Span was marginal $(p=$ $.082)$.

Table 5: Hierarchical regression analysis of Calculation scores. (A) Independent contributions of executive function measures: inhibitory control (Stroop), visuo-spatial working memory (Spatial Span), and cognitive flexibility (TaskSwitch). (B) Executive function and the inclusion of counterintuitive decimal comparisons. 


\begin{tabular}{|c|c|c|c|c|c|}
\hline \multicolumn{6}{|l|}{ Calculation Scores } \\
\hline Model A & B & SE & Beta & $\mathbf{t}$ & $\mathbf{p}$ \\
\hline Intercept & 47.782 & 6.120 & & 7.807 & $<.001$ \\
\hline Age & -1.328 & .267 & -.451 & -4.966 & $<.001$ \\
\hline TaskSwitch RCS & -.700 & .976 & -.078 & -.717 & .475 \\
\hline Spatial Span & .758 & .431 & .182 & 1.758 & .082 \\
\hline Stroop RCS & 4.534 & 2.276 & .214 & 1.992 & .049 \\
\hline \multicolumn{6}{|l|}{ Model B } \\
\hline Intercept & 43.162 & 5.730 & & 7.533 & $<.001$ \\
\hline Age & -1.180 & .248 & -.400 & -4.751 & $<.001$ \\
\hline TaskSwitch RCS & -.076 & .909 & -.009 & -.084 & .933 \\
\hline Spatial Span & .364 & .407 & .087 & .894 & .374 \\
\hline Stroop RCS & 2.452 & 2.149 & .116 & 1.141 & .257 \\
\hline Mixed Inconsistent Decimal & 6.885 & 1.627 & .371 & 4.232 & $<.001$ \\
\hline
\end{tabular}

Next, we examined whether this relationship is maintained in the presence of performance on the Mixed Inconsistent Decimal trials. This model fit the data significantly better $\left(F_{\text {change }}(1,91)=\right.$ $17.91, p<.001$, Table 5, Model B) and explained an additional $11.5 \%$ of the variance. Mixed Inconsistent Decimals was the only significant predictor in the full model $(p<.001)$. Further, none of the EF measures were significant predictors (all $p s>.25$ ).

Finally, to quantify the change of inhibition from a significant to a non-significant predictor of math achievement in the presence of the decimal performance measure, we used mediation analysis. Figure 1B shows that each indirect path in the model is significant and that mediation effect is also significant $(a \times b=.098, p=.042)$, which represents $43.8 \%$ mediation for the direct effect $(c=.214)$.

\subsection{Regression analyses - mathematical specificity}

In a final series of regression analyses, we sought to examine the specificity of the contribution of decimals in explaining the relationship between inhibition and general math achievement. We considered two dimensions of specificity: (1) the mathematical domain of the outcome measure and (2) the mathematical knowledge captured by decimal comparison. For the first dimension, we expect that decimals should explain more of the contribution of inhibitory control to Rational Numbers items than to Advanced Math items. For the second dimension, we reasoned that if performance on Mixed Inconsistent Decimals simply reflects basic mathematical knowledge, including Math Fluency should account for this contribution. However, if decimals performance captures important unique variance for that mathematical domain, it would remain a significant predictor even after including Math Fluency in the model. As our focus in these models was on 
the relative contributions of inhibition, decimals, and fluency, and to avoid overfitting, we did not include Spatial Span or TaskSwitch RCS in these analyses.

After accounting for age, Stroop RCS was a marginal predictor of Rational Number item performance $(p=.067$, Table 6 , Model A), and the overall model was significant $(F(2,94)=$ $11.17, p<.001)$ and explained $17.3 \%$ of the variance. Adding Mixed Inconsistent Decimals to the model increased the fit $\left(F_{\text {change }}(1,93)=12.54, p<.001\right.$, Table 6 , Model B) and explained an additional $9.8 \%$ of variance. Critically, Stroop RCS was no longer a marginal predictor ( $p=$ .399). Finally, adding Math Fluency to this model only modestly improved the fit $\left(F_{\text {change }}(1,92)=\right.$ $3.90, p<.001, .5 \%$ additional variance), and decimals performance remained a significant predictor $(p=.007$, Table 6, Model C).

Table 6: Hierarchical regression analysis of Rational Number items from the Calculation subtest of the WJ-III. (A) Contributions of inhibitory control (Stroop RCS), after accounting for age. (B) Inclusion of counterintuitive decimal comparisons (Mixed Inconsistent Decimal). (C) Inclusion of general arithmetic knowledge (Math Fluency subtest).

\begin{tabular}{lccccc}
\hline Rational Number Items & & & & & \\
\hline Model A & B & SE & Beta & $\mathbf{t}$ & $\mathbf{p}$ \\
\hline Intercept & 10.619 & 2.185 & & 4.861 & $<.001$ \\
Age & -.401 & .094 & -.397 & -4.272 & $<.001$ \\
Stroop RCS & 1.181 & .675 & .163 & 1.750 & .083 \\
\hline Model B & & & & & \\
\hline Intercept & 9.240 & 2.084 & & 4.433 & $<.001$ \\
Age & -.368 & .089 & -.364 & -4.156 & $<.001$ \\
Stroop RCS & .468 & .662 & .064 & .706 & .482 \\
Mixed Inconsistent Decimal & $\mathbf{2 . 1 5 7}$ & .583 & .339 & 3.698 & $<.001$ \\
\hline Model C & & & & & \\
\hline Intercept & & & & & \\
Age & 8.260 & 2.211 & & 3.735 & $<.001$ \\
Stroop RCS & -.358 & .089 & -.354 & -4.031 & $<.001$ \\
Mixed Inconsistent Decimal & 1.897 & .615 & .298 & 3.085 & .003 \\
Math Fluency & .009 & .007 & .121 & 1.290 & .200 \\
\hline & .423 & .661 & .058 & .640 & .524 \\
\hline
\end{tabular}

Finally, we examined this same series of regression models for more advanced items from Calculation that do not require knowledge of rational numbers (Advanced Math items). After 
accounting for age, Stroop RCS was a significant predictor ( $p=.003$, Table 7 , Model A), and the overall model was significant $(F(2,94)=15.03, p<.001)$ and explained $22.6 \%$ of the variance. Adding Mixed Inconsistent Decimals to the model increased the fit $\left(F_{\text {change }}(1,93)=4.348, p=\right.$ .040 , Table 7 , Model B) and explained an additional $2.7 \%$ of the variance, but Stroop RCS remained a significant predictor $(p=.021)$. Further, adding Math Fluency to this model significantly improved the fit $\left(F_{\text {change }}(1,92)=4.860, p=.030,3.0 \%\right.$ extra variance explained $)$, and critically Mixed Inconsistent Decimal performance was no longer a significant predictor $(p=$ .201, Table 7, Model C).

Table 7: Hierarchical regression analysis of Advanced Math items from the Calculation subtest of the WJ-III. (A) Contributions of inhibitory control (Stroop RCS), after accounting for age. (B) Inclusion of counterintuitive decimal comparisons (Mixed Inconsistent Decimal). (C) Inclusion of general arithmetic knowledge (Math Fluency subtest).

\begin{tabular}{|c|c|c|c|c|c|}
\hline \multicolumn{6}{|l|}{ Advance Math Items } \\
\hline Model A & B & SE & Beta & $\mathbf{t}$ & $\mathbf{p}$ \\
\hline Intercept & 7.445 & 2.212 & & 3.526 & $<.001$ \\
\hline Age & -.402 & .091 & -.397 & -4.423 & $<.001$ \\
\hline Stroop RCS & 1.980 & .653 & .273 & 3.034 & .003 \\
\hline \multicolumn{6}{|l|}{ Model B } \\
\hline Intercept & 6.659 & 2.109 & & 3.157 & .002 \\
\hline Age & -.383 & .090 & -.379 & -4.269 & $<.001$ \\
\hline Stroop RCS & 1.573 & .670 & .217 & 2.346 & .021 \\
\hline Mixed Inconsistent Decimal & 1.230 & .590 & .193 & 2.085 & .040 \\
\hline \multicolumn{6}{|l|}{ Model C } \\
\hline Intercept & 4.993 & 2.200 & & 2.269 & .026 \\
\hline Age & -.364 & .088 & -.361 & -4.129 & $<.001$ \\
\hline Stroop RCS & 1.497 & .658 & .206 & 2.276 & .025 \\
\hline Mixed Inconsistent Decimal & .789 & .612 & .124 & 1.289 & .201 \\
\hline Math Fluency & .016 & .007 & .206 & 2.205 & .030 \\
\hline
\end{tabular}

Considering these models from a mediation perspective, decimal performance mediated $60.7 \%$ of the relationship between inhibition and rational number performance, but only $15.3 \%$ of the relationship with advanced math performance. Conversely, fluency explained only $12.1 \%$ of the influence of decimals on rational numbers, but $35.8 \%$ of its contribution on advanced math. 


\section{Discussion}

This study investigated the relationship between EF and academic achievement by focusing on a theoretically motivated connection between inhibitory control and a precursor mathematical skill: the processing of rational numbers in the context of conflicting whole number knowledge. First, we established that inhibitory control is an independent predictor of math achievement in young adults. Then, we determined that performance on a conflicting decimal comparison task explained that relationship. Finally, we found that the contribution of decimal understanding to math achievement was concentrated in the rational number items of this measure. These results are consistent with our framework proposing that mathematical precursor skills can explain the relationship between specific executive functions and specific domains of mathematical achievement.

\subsection{Executive function contributions to math achievement in young adults}

The relations between EF and academic achievement has received considerable attention in recent years, yet most of this work is concentrated in the preschool and early elementary school age children (Bull, Espy, \& Wiebe, 2008; Bull \& Lee, 2014; Clark, Pritchard, \& Woodward, 2010; Lee \& Bull, 2016). Far less is known about the contributions of these skills to the mathematical content taught in secondary school and even less at the college level. Amongst EF measures, the consensus emerging from meta-analyses is a robust contribution of working memory across mathematical domains and age groups (Peng et al., 2016), with less consistent effects for inhibitory control and cognitive flexibility (Friso-van den Bos et al., 2013; Lee \& Lee, 2019). In the current study, measures of all three executive functions were associated with performance on the untimed, progressive math measure, while there were no relations of EF with the timed math fluency measure. This result points towards a continued role for EF in more advanced mathematics. It also suggests that simpler skills like arithmetic no longer depend on EF in adulthood, consistent with the findings of a shift from effortful counting strategies to more memory based retrieval approaches in early elementary school (Qin et al., 2014; Roussel, Fayol, \& Barrouillet, 2002; Siegler, 1996).

Of the EF measures, only inhibition was a significant independent predictor of Calculation performance, with a marginal effect for working memory and no effect for cognitive flexibility. In contrast to the well-established measures of working memory and inhibitory control (Corsi block tapping and color-word Stroop, respectively), our measure of cognitive flexibility was newly developed for the ACE platform (Younger et al., 2021, April 20), and did not display the expected pattern of switching costs. The lack of reliability in this measure may have contributed to the null effects found here, and point to the need to examine other measures of cognitive flexibility, such as the Wisconsin Card Sort Task (Grant \& Berg, 1948) and the Alternative Uses Test (Guilford, 1967) to fully gauge the contribution of this construct to more advanced mathematical understanding. Building on our framework, which emphasizes the need for theoretical relations between constructs, the contributions of cognitively flexibility may be more apparent in some mathematical domains and instructional approaches than others. For example, productive failure, the curriculum approach where students generate solutions to challenging problems before receiving relevant instruction, may draw especially on cognitive flexibility capacities (Kapur, 2008; Schwartz \& Martin, 2004).

Beyond the canonical EF measures, studies of higher cognitive capacities must also consider the contributions of lower-level capacities like processing speed, which have been related to 
academic achievement and executive functioning (Gordon, Smith-Spark, Newton, \& Henry, 2020; Peterson et al., 2017). While we lacked a full dataset to assess the contribution of basic reaction time, among the remaining participants, we found no relationship of this task with either math or inhibition measures, suggesting processing speed differences are not driving the results reported here.

Our finding of the prominent role for inhibitory control among college students suggests this capacity may become especially important as the sophistication of mathematical concepts increases. As the number of mathematical domains students must master expands in secondary school, inhibitory control may be increasingly recruited to identify and apply the appropriate conceptual construct for a given problem (Brookman-Byrne, Mareschal, Tolmie, \& Dumontheil, 2018). In the current context, the significant, independent contribution of inhibitory control on general math achievement establishes the direct pathway between these constructs, setting the stage for identifying specific precursor skills that help explain that relationship.

\subsection{Identifying an appropriate precursor skill in decimal comparison}

The precursor skill that we considered to potentially mediate the relationship between inhibitory control and math achievement was susceptibility to the string length congruity effect (also called the semantic interference effect) in decimal comparison. We focused on this effect as prior work suggested that interference from whole number knowledge results in reduced performance on problems where the number of digits is inconsistent with the correct response (Huber et al., 2014; Varma \& Karl, 2013). As expected, performance was slower and less accurate when the larger number had fewer digits, relative to problems when the larger number had more digits. Our experimental design also enabled examination of other interference and facilitation effects in decimal comparison stimuli. Potentially, these effects could also act as precursor skills, which could relate executive functions to broader mathematical knowledge. To assess the unit decade compatibility effect (Nuerk et al., 2011; Varma \& Karl, 2013), and the zero facilitation effect (Varma \& Karl, 2013), we included the Uniform and Zero conditions, respectively. Although we found no evidence for these effects in performance, in each case, the Inconsistent condition had a modest correlation with Calculation performance. However, these associations were weaker than the relationship between our target skill (Mixed Inconsistent performance) and Calculation. Moreover, neither the Uniform nor the Zero condition showed any relationship to inhibitory control. Together, these results confirm the robustness of the string length congruity effect among decimal comparison manipulations, and suggest that it is a promising target for examining the role of inhibitory control on rational number processing and overall math skills.

\subsection{Types of inhibitory control and their contributions to rational number processing}

Inhibitory control is not a unitary concept, and a common division contrasts semantic inhibition the capacity to resolve interference from acquired knowledge - with response inhibition - the capacity to withhold prepotent behavioral responses. Establishing which construct most strongly relates to rational number understanding is potentially important for identifying diagnostic predictors of future rational number learning difficulties. Further, if semantic inhibition were the more consistent predictor of rational number outcomes, it would lend support to the interpretation that learned knowledge (i.e., whole number knowledge) is the source of interference in rational number tasks. Semantic inhibition is often assessed via Stroop tasks, which use bivalent stimuli and require participants to attend to one dimension and ignore information from the other. Evidence for the importance of semantic inhibition in rational number 
processing comes from Gómez et al. (2015), who find that children with poorer performance on a Numerical Stroop task also struggle with inconsistent fraction comparison. While Avgerinou and Tolmie (2019) report that when children are comparing conflicting fractions and decimals embedded in low-load word problems, performance correlates with a Go/No-Go task (measure of response inhibition), in a high-load situation, they found that Animal Stroop scores became a critical predictor.

While these results pointed towards the importance of semantic inhibition for rational number understanding, two recent studies have found that the Hearts and Flowers task, a developmentally appropriate measure of response inhibition (Wright \& Diamond, 2014), also relates to rational number outcomes in children. Abreu-Mendoza, Coulanges, Ali, Powell, and Rosenberg-Lee (2020) found that better inhibitory control was related to better performance on a discrete proportional reasoning task, specifically when the number of segments conflicted with the correct response. Ren and Gunderson (2021) report that children with better inhibitory control displayed less use of an incorrect whole number strategy during a decimal comparison, training task, and also at post-test. By contrast, one study to date did not find a role for inhibitory control in either symbolic fraction understanding and nonsymbolic proportional reasoning, in college students (Matthews et al., 2016). Notably, in that study, they did not separately examine conflicting vs. compatible stimuli in either format, potentially washing out the specific contribution of inhibitory control to the inconsistent stimuli. Further, the inhibition measure employed, the Flanker task, is considered by some (Lee \& Lee, 2019) as neither semantic or response inhibition, but rather a measure of visual distraction suppression.

In the current study, we employed the color-word Stroop task, which requires inhibiting prior knowledge of the meanings of symbols strings in order to correctly identify the font color of a presented color word. We selected this measure based on the earlier literature (Avgerinou \& Tolmie, 2019; Gómez et al., 2015) pointing to the importance of semantic inhibition in rational number outcomes. Our finding that color-word Stroop performance predicts performance specifically on conflicting decimal comparison (Indirect Pathway-1) extends the prior literature to demonstrate that semantic measures of inhibitory control that do not require any knowledge of numerical or physical magnitude (as found in Numerical and Animal Stroop tasks) also relate to rational number understanding. However, given the growing evidence that response inhibition also contributes, it is necessary to design studies that collect multiple measures of inhibitory control within the same subjects. This approach is required to establish if semantic, response and visual distractor suppression inhibition contribute to conflicting rational performance, and whether they do so independently.

\subsection{Decimal comparison and overall math skills}

Relations between numerical comparison tasks and math skills are well-established (Halberda et al., 2008; Schneider et al., 2017), and more recently, researchers have asked whether rational number comparisons skills might also relate to overall math skills. A handful of fraction studies find that associations of comparison performance with measures of general mathematics achievement. For example Gómez et al. (2015) related performance on incongruent fraction comparison to classroom math measures, and Matthews et al. (2016) found that comparing line lengths and dot proportions predicted math skills in college students.

We found that performance on the Mixed Inconsistent trials of the decimal comparison task correlated with overall math achievement (Indirect Pathway-2). This result is, to our knowledge, 
the first demonstration of a relationship between decimal comparison and overall math skills in adults, although similar effects have been found in children (Resnick, 2019). Notably, we did not find any relationship between the math assessments and performance on the Mixed Consistent trials, arguing against the possibility that the relationship found for Mixed Inconsistent reflects general number knowledge tapped by any form of decimal comparison. However, the high accuracy and low variance in the Mixed Consistent condition suggest ceiling effects may be driving this null result. Further, they point towards the need for studies to include both whole number and decimal comparison tasks to establish whether the contribution of processing conflicting rational numbers to math achievement is independent of general numerical understanding (Van Hoof, Verschaffel, \& Van Dooren, 2017).

Given prior work relating fraction comparison and estimation to general math skills, we can ask whether both fraction and decimal comparison of conflicting rational number problems index the same underlying capacity, that is, the ability to appropriately process rational number magnitude in the face of interference from whole number information. If so, we would expect both factors to contribute to later math skills, as found for fraction and decimal number line placement and algebra understanding (DeWolf et al., 2015; Siegler et al., 2012). We would also expect them to correlate with each other. One study to date has looked at correlation between these measures, in terms of distance effects in a comparison to a fixed value. DeWolf and colleagues (2014) report that distance effects for decimals were more similar to those for integers than for fractions. However, this study did not systematically vary interference from whole numbers in the decimal stimuli nor did it directly assess individual differences in this capacity across a large sample. (Note, Avgerinou and Tolmie (2019) collected both measures but then averaged them together for subsequent analyses.)

One complicating factor for fractions is that comparison tasks are rife with strategy variation, including componential strategies like selecting the fraction with the largest numerator or the largest denominator and more sophisticated approaches such as the gap strategy of selecting the fraction with the smaller gap between its numerator and denominator. Thus, abstracting a single index of fraction understanding from comparison tasks may be challenging without considering strategy information (Braithwaite, Pyke, \& Siegler, 2017). Decimal comparison is not without strategy variation (Ren \& Gunderson, 2019; Resnick et al., 1989). A further complication is that inconsistent decimals comparison involves interference from both numerical values of stimuli and also their physical length, as demonstrated by recent negative priming studies (Roell, Viarouge, Hilscher, Houde, \& Borst, 2019; Roell, Viarouge, Houde, et al., 2019),. Collecting data from the same participants completing both decimals and fraction comparison tasks (that manipulate whole number interference) is needed to determine if fraction and decimal precursor skills make independent contributions to math achievement, or if both reflect an underlying construct of "rational number understanding".

Nevertheless, some evidence already points towards a foundational role for decimal knowledge relative to fractions. For example, Van Hoof et al. (2018) documented trajectories through rational number knowledge, and finds that rational number ordering/comparison is usually mastered first, followed by understanding of arithmetic operations (i.e., that multiplication does not always lead to a larger number), and finally density concepts (i.e., the infinity of rational numbers between any two numbers). In each of these domains, proficiency with decimals precedes fraction understanding, suggesting that understanding decimal magnitude is the first rational number skill to fully develop. The relative simplicity of decimals, which maintain much of 
the base-ten place value system of whole numbers, may facilitate its earlier emergence, despite typically being taught after fractions (Tian \& Siegler, 2018).

If conflicting decimal comparison is acting as precursor skill for higher mathematical concepts, as we found in the current study, then we can ask what foundational ability does this task index? Assuming that the primary source of difficulty in this task is interference from one's prior whole number knowledge that more digits indicate a larger value (Huber et al., 2014), then greater proficiency in this task may capture decoupling the association between the number of digits and magnitude. This aspect of whole number knowledge also interferes with fraction comparison, as children will judge double-digit fractions as larger than their equivalent singledigit fraction (e.g., 20/25 > 4/5) (Braithwaite \& Siegler, 2018). Decimal comparison difficulties have also been attributed to interference from the whole number referents (i.e., ignoring the decimal points) (Varma \& Karl, 2013). Thus, proficiency on this task could also represent breaking the association between number symbols and magnitude, which could contribute more generally to fraction comparison, where magnitude information must be gleaned holistically, rather than from the individual components (Meert, Gregoire, \& Noel, 2009). In the realm of rational arithmetic, a better sense of the magnitude of numbers, regardless of notation, could minimize nonsensical responses (like adding numerators and denominators, or misplaced decimal points) (Braithwaite et al., 2017; Resnick et al., 1989). Thus, grasping conflicting decimals requires flexibly mapping between number symbols and magnitude, expanding one's conceptual understanding of abstract number classes (Carey, 2011; Rosenberg-Lee, 2021, March 24).

\subsection{Relating inhibitory control to math achievement through decimal processing}

Having found evidence that inhibition independently contributes to math achievement, and that decimal comparison relates to both inhibitory control and math achievement, we next asked whether decimal comparison could explain some of the relationship between an executive function measure and a math outcome. We found that performance on conflicting decimals accounted for almost half of the relationship between inhibition and general math achievement. The remainder of that relationship could be due to the requirement of inhibition during the test itself (Bascandziev et al., 2018) and any number of other precursor skills we have not measured, whose acquisition may have also be supported by inhibitory control. Other numerical domains with counterintuitive properties, such as integers, are promising targets for precursor content that requires inhibition to learn, and supports later mathematical understanding (Blair, Rosenberg-Lee, Tsang, Schwartz, \& Menon, 2012; Brookman-Byrne et al., 2018; Varma \& Schwartz, 2011).

The current pattern of results is consistent with our starting premise that single time-point correlations between executive functions and general math skills reflect both a direct role for EF in the execution of problem solving strategies and an indirect role supporting the acquisition of precursor mathematical content. While we cannot measure these contributions directly with our current design, we note that the relative contributions of these factors likely change over time. For example, in the realm of arithmetic, EFs did not predict fluency in our college-aged sample, despite their well-established contribution to arithmetic fluency in childhood (Geary, 2003; Meyer, Salimpoor, Wu, Geary, \& Menon, 2010; Peng et al., 2016). Thus, EFs likely supported the initial acquisition of arithmetic skills, but by young adulthood, everyone has mastered these skills and can generally solve single-digit problems without relying on EFs. Still, this finding does not mean that EFs should be uncorrelated with later math achievement. Rather, earlier mastery 
of arithmetic could facilitate better learning of later content, which thus contributes to relations between EF and higher mathematics. Consistent with this proposal is our finding that arithmetic fluency mediated the relationship between inhibition and advanced math items. Longitudinal designs, which focus on the prediction of learning gains, can help disentangle the contribution of EF to performance in the moment versus future learning.

More broadly, we offer these methods as an alternative to the usual approaches to investigating precursor skills and math cognition. Researchers typically aim to support the finding of a correlation result by showing that it is robust to the inclusion of measures of executive function (Hiniker, Rosenberg-Lee, \& Menon, 2016; Matthews et al., 2016; Siegler et al., 2012), or to challenge a finding by showing that including EF eliminates an association (Gilmore et al., 2013). (Or, less frequently, they aim to show that math ability explains the relationship between EF and a precursor skill (Gómez et al., 2015).) Fundamentally, these approaches assume there are more interesting effects that must be shown to be robust to the inclusion of less interesting factors. Rather than consider some factors as nuisances to be controlled for, we suggest instead that considering plausible pathways to connect factors may be more fruitful. Here, we explore the proposal that correlations between executive functions and overall math skills arise, in part, because of the support those EFs provide in acquiring specific precursor skills, which in turn support later mathematical content (Figure 1A).

This perspective also affords research that can eventually leverage these associations into classroom-applicable insights via training studies (Rosenberg-Lee, 2018). For example, if inhibitory control specifically contributes to understanding conflicting decimals, which then supports acquisition of later mathematical content, then we would expect specific training in processing these decimals to be more effective in boosting math outcomes than a more general inhibitory control training program. By the same token, we would expect that training programs which emphasize conflicting aspects of rational numbers to be more effective than those that emphasize similarities between whole and rational numbers (Rosenberg-Lee, 2021, March 24). By contrast, if relations of precursor skills to math achievement simply reflect the role of EF on both measures, then we should not expect to see any benefits for shoring up a particular challenging content domain. If these predictions regarding the effectiveness of training decimal precursors skills are borne out, our approach could also be applied in other content domains, by identifying bottlenecks in precursor skills engendered by executive function deficits, and then developing education programs to target these pressure points.

\subsection{Fractionating math achievement to assess the specificity of decimal comparison.}

To understand further the contribution of inhibitory control to math achievement via the acquisition of precursor skills, such as conflicting decimal comparison, we decomposed the Calculation subtest into Rational Number items and Advanced Math items. We found that Inconsistent Decimal performance mediated much more of the relationship of Stroop with rational numbers than with advanced math. While the contribution of decimal comparison to rational numbers may not be surprising on the surface, it should be noted that we purposely excluded decimal arithmetic items in the sub-measure. This finding fits with the suggestion that decimal comparison taps vital knowledge for rational number understanding, that is, the decoupling of associations between notation and numerical magnitude.

Further evidence for the specificity of the contribution of decimal comparison to rational number understanding comes from our analyses of Math Fluency. While this measure was significantly 
related to conflicting decimal performance, it did not predict rational number items. Interestingly, Math Fluency did contribute to the advanced math items, and it mediated the contribution of conflicting decimals to those items. It is notable that inhibitory control had a smaller contribution to rational number items than advanced math items. This result may reflect the small number of items in this latter sub-measure (Rational Number: 7 items, vs. Advanced Math: 11 items), in which case a more comprehensive measure of rational number understanding (Kainulainen et al., 2017; Van Hoof et al., 2018) might reveal a more robust relationship. Alternatively, inhibitory control may be most crucial in the acquisition of new, counterintuitive knowledge and the specific contribution of individual differences in this capacity may be washed out over time. Individual difference studies in children, when they are first learning about decimals and fractions (typically in late elementary school), are needed to fully capture the role of inhibition in acquiring rational number understanding.

\section{Acknowledgements}

The authors wish to thank Jessica W. Younger for her efforts in compiling the Adaptive Cognitive Evaluation (ACE) methods. This research was supported in part by NSF Award SMA1540854 (MU and AG). 
Abreu-Mendoza, R. A., Coulanges, L., Ali, K., Powell, A. B., \& Rosenberg-Lee, M. (2020). Children's discrete proportional reasoning is related to inhibitory control and enhanced by priming continuous representations. J Exp Child Psychol, 199, 104931. doi:10.1016/j.jecp.2020.104931

Avgerinou, V. A., \& Tolmie, A. (2019). Inhibition and cognitive load in fractions and decimals. The British journal of educational psychology. doi:10.1111/bjep.12321

Bascandziev, I., Tardiff, N., Zaitchik, D., \& Carey, S. (2018). The role of domain-general cognitive resources in children's construction of a vitalist theory of biology. Cogn Psychol, 104, 1-28. doi:10.1016/j.cogpsych.2018.03.002

Blair, K. P., Rosenberg-Lee, M., Tsang, J. M., Schwartz, D. L., \& Menon, V. (2012). Beyond natural numbers: negative number representation in parietal cortex. Front Hum Neurosci, 6(7), 1-15.

Bonato, M., Fabbri, S., Umiltà, C., \& Zorzi, M. (2007). The mental representation of numerical fractions: Real or integer? Journal of Experimental Psychology: Human Perception and Performance, 33(6), 1410-1419. doi:10.1037/0096-1523.33.6.1410

Braithwaite, D. W., Pyke, A. A., \& Siegler, R. S. (2017). A computational model of fraction arithmetic. Psychol Rev, 124(5), 603-625. doi:10.1037/rev0000072

Braithwaite, D. W., \& Siegler, R. S. (2018). Developmental changes in the whole number bias. Developmental Science, 21(2), 13. doi:10.1111/desc.12541

Brookman-Byrne, A., Mareschal, D., Tolmie, A. K., \& Dumontheil, I. (2018). Inhibitory control and counterintuitive science and maths reasoning in adolescence. PLoS ONE, 13(6). doi:10.1371/journal.pone.0198973

Bull, R., Espy, K. A., \& Wiebe, S. A. (2008). Short-term memory, working memory, and executive functioning in preschoolers: Longitudinal predictors of mathematical achievement at age 7 years. Developmental Neuropsychology, 33(3), 205-228. doi:10.1080/87565640801982312

Bull, R., \& Lee, K. (2014). Executive Functioning and Mathematics Achievement. Child Development Perspectives, 8(1), 36-41. doi:10.1111/cdep.12059

Carey, S. (2011). Precis of 'The Origin of Concepts'. Behav Brain Sci, 34(3), 113-124; discussion 124-162. doi:10.1017/S0140525X10000919

Clark, C. A. C., Pritchard, V. E., \& Woodward, L. J. (2010). Preschool executive functioning abilities predict early mathematics achievement. Developmental Psychology, 46(5), 1176-1191. doi:10.1037/a0019672

Constantinidis, C., \& Luna, B. (2019). Neural Substrates of Inhibitory Control Maturation in Adolescence. Trends in Neurosciences, 42(9), 604-616. doi:10.1016/j.tins.2019.07.004

Corsi, P. (1972). HUMAN MEMORY AND THE MEDIAL TEMPORAL REGION OF THE BRAIN. In: ProQuest Dissertations Publishing.

De Smedt, B., Noël, M.-P., Gilmore, C., \& Ansari, D. (2013). How do symbolic and non-symbolic numerical magnitude processing skills relate to individual differences in children's mathematical skills? A review of evidence from brain and behavior. Trends in Neuroscience and Education, 2(2), 48-55. doi:10.1016/j.tine.2013.06.001

DeWolf, M., Bassok, M., \& Holyoak, K. J. (2015). From rational numbers to algebra: separable contributions of decimal magnitude and relational understanding of fractions. $J$ Exp Child Psychol, 133, 72-84. doi:10.1016/j.jecp.2015.01.013

DeWolf, M., Grounds, M. A., Bassok, M., \& Holyoak, K. J. (2014). Magnitude comparison with different types of rational numbers. J Exp Psychol Hum Percept Perform, 40(1), 71-82. doi:10.1037/a0032916

Diamond, A. (2013). Executive functions. Annu Rev Psychol, 64, 135-168. doi:10.1146/annurevpsych-113011-143750 
Friso-van den Bos, I., van der Ven, S. H. G., Kroesbergen, E. H., \& van Luit, J. E. H. (2013). Working memory and mathematics in primary school children: A meta-analysis. Educational Research Review, 10, 29-44. doi:10.1016/j.edurev.2013.05.003

Geary, D. C. (2003). Learning disabilities in arithmetic: Problem-solving differences and cognitive deficits In H. L. Swanson \& K. R. Harris (Eds.), Graham, Steve; Ed; Handbook of learning disabilities (pp. 199-212). New York, NY: Guilford Press.

Gersten, R., \& Chard, D. (1999). Number sense: rethinking arithmetic instruction for students with mathematical disabilities. Journal of Special Education, 33(1), 18-28.

Gilmore, C., Attridge, N., Clayton, S., Cragg, L., Johnson, S., Marlow, N., Simms, V., \& Inglis, M. (2013). Individual Differences in Inhibitory Control, Not Non-Verbal Number Acuity, Correlate with Mathematics Achievement. PLoS ONE, 8(6). doi:DOI 10.1371/journal.pone.0067374

Gómez, D. M., Jiménez, A., Bobadilla, R., Reyes, C., \& Dartnell, P. (2015). The effect of inhibitory control on general mathematics achievement and fraction comparison in middle school children. Zdm, 47(5), 801-811. doi:10.1007/s11858-015-0685-4

Gordon, R., Smith-Spark, J. H., Newton, E. J., \& Henry, L. A. (2020). Working memory and high-level cognition in children: An analysis of timing and accuracy in complex span tasks. J Exp Child Psychol, 191, 104736. doi:10.1016/j.jecp.2019.104736

Grant, D. A., \& Berg, E. (1948). A behavioral analysis of degree of reinforcement and ease of shifting to new responses in a Weigl-type card-sorting problem. Journal of Experimental Psychology, 38(4), 404-411. doi:10.1037/h0059831

Guilford, J. P. (1967). Creativity: Yesterday, Today and Tomorrow. The Journal of Creative Behavior, 1(1), 3-14. doi:10.1002/j.2162-6057.1967.tb00002.x

Halberda, J., Mazzocco, M. M. M., \& Feigenson, L. (2008). Individual differences in non-verbal number acuity correlate with maths achievement Nature, 455(7213), 665-U662.

Retrieved from <Go to ISI>://000259639700047

Hedge, C., Powell, G., \& Sumner, P. (2018). The reliability paradox: Why robust cognitive tasks do not produce reliable individual differences. Behav Res Methods, 50(3), 1166-1186. doi:10.3758/s13428-017-0935-1

Hiniker, A., Rosenberg-Lee, M., \& Menon, V. (2016). Distinctive Role of Symbolic Number Sense in Mediating the Mathematical Abilities of Children with Autism. J Autism Dev Disord, 46(4), 1268-1281. doi:10.1007/s10803-015-2666-4

Huber, S., Klein, E., Willmes, K., Nuerk, H. C., \& Moeller, K. (2014). Decimal fraction representations are not distinct from natural number representations - evidence from a combined eye-tracking and computational modeling approach. Front Hum Neurosci, 8, 172. doi:10.3389/fnhum.2014.00172

Ishihara, S. (1972). Tests for Colour-Blindness. Tokyo, Japan: Kanehara Shuppan Co.

Kainulainen, M., McMullen, J., \& Lehtinen, E. (2017). Early Developmental Trajectories Toward Concepts of Rational Numbers. Cognition and Instruction, 35(1), 4-19. doi:10.1080/07370008.2016.1251287

Kapur, M. (2008). Productive Failure. Cognition and Instruction, 26(3), 379-424. doi:10.1080/07370000802212669

Kenny, D. A., Korchmaros, J. D., \& Bolger, N. (2003). Lower level mediation in multilevel models. Psychological methods, 8(2), 115-128. doi:10.1037/1082-989x.8.2.115

Lee, K., \& Bull, R. (2016). Developmental Changes in Working Memory, Updating, and Math Achievement. Journal of Educational Psychology, 108(6), 869-882. doi:10.1037/edu0000090

Lee, K., \& Lee, H. W. (2019). Inhibition and Mathematical Performance: Poorly Correlated, Poorly Measured, or Poorly Matched? Child Development Perspectives, 13(1), 28-33. doi:10.1111/cdep.12304 
Mason, L., \& Zaccoletti, S. (2020). Inhibition and Conceptual Learning in Science: a Review of Studies. Educational Psychology Review. doi:10.1007/s10648-020-09529-x

Matthews, P. G., Lewis, M. R., \& Hubbard, E. M. (2016). Individual Differences in Nonsymbolic Ratio Processing Predict Symbolic Math Performance. Psychological Science, 27(2), 191-202. doi:10.1177/0956797615617799

Mead, L. A., Mayer, A. R., Bobholz, J. A., Woodley, S. J., Cunningham, J. M., Hammeke, T. A., \& Rao, S. M. (2002). Neural basis of the Stroop interference task: Response competition or selective attention? Journal of the International Neuropsychological Society, 8(6), 735-742. doi:10.1017/S1355617702860015

Meert, G., Gregoire, J., \& Noel, M. P. (2009). Rational numbers: componential versus holistic representation of fractions in a magnitude comparison task. $Q \mathrm{~J}$ Exp Psychol (Colchester), 62(8), 1598-1616. doi:10.1080/17470210802511162

Melby-Lervag, M., \& Hulme, C. (2013). Is working memory training effective? A meta-analytic review. Developmental Psychology, 49(2), 270-291. doi:10.1037/a0028228

Meyer, M. L., Salimpoor, V. N., Wu, S. S., Geary, D. C., \& Menon, V. (2010). Differential contribution of specific working memory components to mathematics achievement in 2nd and 3rd graders. Learning and Individual Differences, 20(2), 101-109. doi:10.1016/j.lindif.2009.08.004

National Mathematics Advisory Panel. (2008). Foundations for success: The final report of the National Mathematics Advisory Panel. Retrieved from Washington, DC:

Ni, Y., \& Zhou, Y.-D. (2005). Teaching and Learning Fraction and Rational Numbers: The Origins and Implications of Whole Number Bias. Educational Psychologist, 40(1), 27-52. doi:10.1207/s15326985ep4001_3

Nuerk, H.-C., Moeller, K., Klein, E., Willmes, K., \& Fischer, M. H. (2011). Extending the Mental Number Line. Zeitschrift für Psychologie, 219(1), 3-22. doi:10.1027/2151-2604/a000041

Nuerk, H.-C., Weger, U., \& Willmes, K. (2001). Decade breaks in the mental number line? Putting the tens and units back in different bins. Cognition, 82(1), B25-B33. doi:10.1016/S0010-0277(01)00142-1

Obersteiner, A., \& Tumpek, C. (2016). Measuring fraction comparison strategies with eyetracking. Zdm-the International Journal on Mathematics Education, 48(3), 255-266. doi:10.1007/s11858-015-0742-z

Obersteiner, A., Van Dooren, W., Van Hoof, J., \& Verschaffel, L. (2013). The natural number bias and magnitude representation in fraction comparison by expert mathematicians. Learning and Instruction, 28, 64-72. doi:10.1016/j.learninstruc.2013.05.003

Peirce, J. W. (2007). PsychoPy--Psychophysics software in Python. J Neurosci Methods, 162(12), 8-13. doi:10.1016/j.jneumeth.2006.11.017

Peng, P., Namkung, J., Barnes, M., \& Sun, C. (2016). A Meta-Analysis of Mathematics and Working Memory: Moderating Effects of Working Memory Domain, Type of Mathematics Skill, and Sample Characteristics. Journal of Educational Psychology, 108(4), 455-473. doi:10.1037/edu0000079

Peterson, R. L., Boada, R., McGrath, L. M., Willcutt, E. G., Olson, R. K., \& Pennington, B. F. (2017). Cognitive Prediction of Reading, Math, and Attention: Shared and Unique Influences. Journal of Learning Disabilities, 50(4), 408-421. doi:10.1177/0022219415618500

Qin, S. Z., Cho, S., Chen, T. W., Rosenberg-Lee, M., Geary, D. C., \& Menon, V. (2014). Hippocampal-neocortical functional reorganization underlies children's cognitive development. Nature Neuroscience, 17(9), 1263-1269. doi:Doi 10.1038/Nn.3788

$\mathrm{R}$ Core Team. (2018). R: A language and environment for statistical computing. Retrieved from https://www.R-project.org 
Ren, K., \& Gunderson, E. A. (2019). Malleability of Whole-Number and Fraction Biases in Decimal Comparison. Developmental Psychology, 55(11), 2263-2274. doi:10.1037/dev0000797

Ren, K. X., \& Gunderson, E. A. (2021). The dynamic nature of children's strategy use after receiving accuracy feedback in decimal comparisons. Journal of Experimental Child Psychology, 202. doi:10.1016/j.jecp.2020.105015

Resnick, I., Rinne, L., Barbieri, C., \& Jordan, N. C. (2019). Children's reasoning about decimals and its relation to fraction learning and mathematics achievement. Journal of Educational Psychology, 111(4), 604-618. doi:10.1037/edu0000309

Resnick, L., Nesher, P., Leonard, F., Magone, M., Omanson, S., \& Peled, I. (1989). Conceptual Bases of Arithmetic Errors: The Case of Decimal Fractions. Journal for Research in Mathematics Education, 20(1), 8-27. doi:10.2307/749095

Roell, M., Viarouge, A., Hilscher, E., Houde, O., \& Borst, G. (2019). Evidence for a visuospatial bias in decimal number comparison in adolescents and in adults. Scientific reports, 9(1), 14770. doi:10.1038/s41598-019-51392-6

Roell, M., Viarouge, A., Houde, O., \& Borst, G. (2017). Inhibitory control and decimal number comparison in school-aged children. PLOS ONE, 12(11), e0188276. doi:10.1371/journal.pone.0188276

Roell, M., Viarouge, A., Houde, O., \& Borst, G. (2019). Inhibition of the whole number bias in decimal number comparison: A developmental negative priming study. J Exp Child Psychol, 177, 240-247. doi:10.1016/j.jecp.2018.08.010

Rosenberg-Lee, M. (2018). Training Studies: An Experimental Design to Advance Educational Neuroscience. Mind, brain and education : the official journal of the International Mind, Brain, and Education Society., 12(1), 12-22. doi:10.1111/mbe.12166

Rosenberg-Lee, M. (2021, March 24). Probing the neural basis rational number difficulties: the role of inhibitory control and magnitude processing. In A. Henik \& W. Fias (Eds.), Learning and Education in Numerical Cognition: Elsevier.

Rossi, S., Vidal, J., Letang, M., Houdé, O., \& Borst, G. (2019). Adolescents and adults need inhibitory control to compare fractions. Journal of Numerical Cognition, 5(3), 314-336. doi:10.5964/jnc.v5i3.197

Roussel, J. L., Fayol, M., \& Barrouillet, P. (2002). Procedural vs. direct retrieval strategies in arithmetic: A comparison between additive and multiplicative problem solving. European Journal of Cognitive Psychology, 14(1), 61-104. Retrieved from < Go to ISI>://000173108300005

Sasanguie, D., Lyons, I. M., De Smedt, B., \& Reynvoet, B. (2017). Unpacking symbolic number comparison and its relation with arithmetic in adults. Cognition, 165, 26-38. doi:10.1016/j.cognition.2017.04.007

Schneider, M., Beeres, K., Coban, L., Merz, S., Susan Schmidt, S., Stricker, J., \& De Smedt, B. (2017). Associations of non-symbolic and symbolic numerical magnitude processing with mathematical competence: a meta-analysis. Developmental Science, 20(3), 1-16. doi:10.1111/desc. 12372

Schwartz, D. L., \& Martin, T. (2004). Inventing to Prepare for Future Learning: The Hidden Efficiency of Encouraging Original Student Production in Statistics Instruction. Cognition and Instruction, 22(2), 129-184. doi:10.1207/s1532690xci2202_1

Siegler, R. S. (1996). A grand theory of development Monographs of the Society for Research in Child Development, 61(1-2), 266-275.

Siegler, R. S., \& Booth, J. L. (2004). Development of Numerical Estimation in Young Children Child Development, 75(2), 428-444. Retrieved from https://onlinelibrary.wiley.com/doi/pdf/10.1111/j.1467-8624.2004.00684.x 
Siegler, R. S., Duncan, G. J., Davis-Kean, P. E., Duckworth, K., Claessens, A., Engel, M., Susperreguy, M. I., \& Chen, M. C. (2012). Early Predictors of High School Mathematics Achievement. Psychological Science, 23(7), 691-697. doi:10.1177/0956797612440101

Siegler, R. S., Thompson, C. A., \& Schneider, M. (2011). An integrated theory of whole number and fractions development. Cognitive Psychology, 62(4), 273-296. doi:10.1016/j.cogpsych.2011.03.001

Stroop, J. (1992). "Reprint of" Studies of Interference in Serial Verbal Reactions: Original work published 1935 (Vol. 18, pp. 643-662). Journal of Experimental Psychology; General, 121(1), 15.

The jamovi project. (2020). jamovi.

Tian, J., \& Siegler, R. S. (2018). Which Type of Rational Numbers Should Students Learn First? Educational Psychology Review, 30(2), 351-372. doi:10.1007/s10648-017-9417-3

Vamvakoussi, X., \& Vosniadou, S. (2004). Understanding the structure of the set of rational numbers: a conceptual change approach. Learning and Instruction, 14(5), 453-467. doi:10.1016/j.learninstruc.2004.06.013

Van Dooren, W., \& Inglis, M. (2015). Inhibitory control in mathematical thinking, learning and problem solving: a survey. Zdm-Mathematics Education, 47(5), 713-721. doi:10.1007/s11858-015-0715-2

Van Hoof, J., Degrande, T., Ceulemans, E., Verschaffel, L., \& Van Dooren, W. (2018). Towards a mathematically more correct understanding of rational numbers: A longitudinal study with upper elementary school learners. Learning and Individual Differences, 61, 99-108. doi:10.1016/j.lindif.2017.11.010

Van Hoof, J., Verschaffel, L., \& Van Dooren, W. (2017). Number sense in the transition from natural to rational numbers. The British journal of educational psychology, 87(1), 43-56. doi:10.1111/bjep.12134

Vandierendonck, A. (2017). A comparison of methods to combine speed and accuracy measures of performance: A rejoinder on the binning procedure. Behav Res Methods, 49(2), 653-673. doi:10.3758/s13428-016-0721-5

Varma, S., \& Karl, S. R. (2013). Understanding decimal proportions: discrete representations, parallel access, and privileged processing of zero. Cogn Psychol, 66(3), 283-301. doi:10.1016/j.cogpsych.2013.01.002

Varma, S., \& Schwartz, D. L. (2011). The mental representation of integers: an abstract-toconcrete shift in the understanding of mathematical concepts. Cognition, 121(3), 363385. doi:10.1016/j.cognition.2011.08.005

Wilkinson, H. R., Smid, C., Morris, S., Farran, E. K., Dumontheil, I., Mayer, S., Tolmie, A., Bell, D., Porayska-Pomsta, K., Holmes, W., Mareschal, D., \& Thomas, M. S. C. (2019). Domain-Specific Inhibitory Control Training to Improve Children's Learning of Counterintuitive Concepts in Mathematics and Science. Journal of Cognitive Enhancement. doi:10.1007/s41465-019-00161-4

Wright, A., \& Diamond, A. (2014). An effect of inhibitory load in children while keeping working memory load constant. Frontiers in psychology, 5, 213. doi:10.3389/fpsyg.2014.00213

Younger, J., O'Laughlin, K. D., Anguera, J., Bunge, S., Ferrer, E., Hoeft, F., McCandliss, B. D., Mishra, J., Rosenberg-Lee, M., Gazzaley, A., \& Uncapher, M. R. (2021, April 20). Development of Executive Function in Middle Childhood: a Large-Scale, In-School, Longitudinal Investigation. doi:https://doi.org/10.31234/osf.io/xf489

Zelazo, P. D., Blair, C., \& Willoughby, M. T. (2017). Executive function : implications for education. Washington, D.C: U.S. Department of Education, Institute of Education Sciences. 


\section{Appendix A}

\begin{tabular}{|c|c|c|c|c|c|}
\hline \multicolumn{2}{|c|}{ Mixed } & \multicolumn{2}{|c|}{ Zero } & \multicolumn{2}{|c|}{ Uniform } \\
\hline Consistent & Inconsistent & Consistent & Inconsistent & Consistent & Inconsistent \\
\hline 0.1 vs. 0.23 & 0.16 vs. 0.4 & 0.10 vs. 0.23 & 0.16 vs. 0.40 & 0.13 vs. 0.24 & 0.17 vs. 0.25 \\
\hline 0.1 vs. 0.26 & 0.18 vs. 0.9 & 0.10 vs. 0.26 & 0.32 vs. 0.50 & 0.13 vs. 0.29 & 0.19 vs. 0.73 \\
\hline 0.1 vs. 0.76 & 0.21 vs. 0.8 & 0.10 vs. 0.76 & 0.18 vs. 0.90 & 0.13 vs. 0.74 & 0.19 vs. 0.83 \\
\hline 0.1 vs. 0.78 & 0.21 vs. 0.9 & 0.10 vs. 0.78 & 0.21 vs. 0.80 & 0.13 vs. 0.89 & 0.19 vs. 0.86 \\
\hline 0.1 vs. 0.83 & 0.23 vs. 0.4 & 0.10 vs. 0.83 & 0.21 vs. 0.90 & 0.21 vs. 0.87 & 0.24 vs. 0.83 \\
\hline 0.2 vs. 0.87 & 0.23 vs. 0.8 & 0.20 vs. 0.87 & 0.23 vs. 0.40 & 0.23 vs. 0.89 & 0.27 vs. 0.91 \\
\hline 0.2 vs. 0.91 & 0.26 vs. 0.3 & 0.20 vs. 0.91 & 0.23 vs. 0.80 & 0.24 vs. 0.95 & 0.29 vs. 0.83 \\
\hline 0.3 vs. 0.91 & 0.26 vs. 0.9 & 0.30 vs. 0.91 & 0.26 vs. 0.30 & 0.31 vs. 0.48 & 0.35 vs. 0.92 \\
\hline 0.3 vs. 0.93 & 0.27 vs. 0.9 & 0.30 vs. 0.93 & 0.26 vs. 0.90 & 0.31 vs. 0.93 & 0.47 vs. 0.75 \\
\hline 0.4 vs. 0.56 & 0.32 vs. 0.5 & 0.40 vs. 0.56 & 0.27 vs. 0.90 & 0.32 vs. 0.54 & 0.48 vs. 0.71 \\
\hline 0.4 vs. 0.62 & 0.36 vs. 0.9 & 0.40 vs. 0.62 & 0.36 vs. 0.90 & 0.35 vs. 0.97 & 0.49 vs. 0.72 \\
\hline 0.6 vs. 0.78 & 0.42 vs. 0.5 & 0.60 vs. 0.78 & 0.42 vs. 0.50 & 0.51 vs. 0.67 & 0.74 vs. 0.93 \\
\hline 0.8 vs. 0.92 & 0.43 vs. 0.6 & 0.80 vs. 0.92 & 0.43 vs. 0.60 & 0.61 vs. 0.78 & 0.79 vs. 0.96 \\
\hline 0.8 vs. 0.97 & 0.67 vs. 0.9 & 0.80 vs. 0.97 & 0.67 vs. 0.90 & 0.73 vs. 0.94 & 0.89 vs. 0.92 \\
\hline
\end{tabular}

